\title{
Combining transgenesis with paratransgenesis to fight malaria
}

2

3 Wei Huang ${ }^{1}$, Joel Vega-Rodriguez ${ }^{2}$, Christopher Kizito ${ }^{1}$, Sung-Jae Cha ${ }^{1}$ and Marcelo

4 Jacobs-Lorena ${ }^{1,3}$

5

6

$7 \quad{ }^{1}$ Department of Molecular Microbiology and Immunology, Malaria Research Institute, Johns

8 Hopkins Bloomberg School of Public Health, Baltimore, MD 21205, USA.

9

$10 \quad{ }^{2}$ Laboratory of Malaria and Vector Research, National Institute of Allergy and Infectious

11 Diseases, National Institutes of Health, 12735 Twinbrook Parkway, Rm 2E20A, Rockville, MD

12 20852, USA.

13

$14{ }^{3}$ To whom correspondence may be addressed. Email: ljacob13@jhu.edu

15

16 Key words: Transgenesis, paratransgenesis, malaria transmission

17 


\section{Significance}

19

20 Malaria kills hundreds of thousand persons yearly. Clearly, new approaches are needed to fight

21 this disease. Two promising approaches are based on the concept of genetically modifying the

22 mosquito to make it a poor vector for the parasite: 1) transgenesis (engineering the mosquito to

23 deliver anti-malarial compounds) and 2) paratransgenesis (engineering mosquito symbiotic

24 bacteria to deliver anti-malarial compounds). The key questions addressed by this manuscript

25 are: which of the two is the most promising approach? And because transgenesis and

26 paratransgenesis are not mutually exclusive, would the combination of both be the most effective

27 strategy? Our results argue for the combination of the two, showing the additive impact that

28 these two strategies may have in controlling malaria transmission in the field. 
Abstract

31 Malaria is among the deadliest infectious diseases and Plasmodium, the causative agent, needs to

32 complete a complex development cycle in its vector mosquito for transmission to occur. Two

33 promising strategies to curb transmission are transgenesis, consisting of genetically engineering

34 mosquitoes to express anti-malarial effector molecules and paratransgenesis, consisting of introducing into the mosquito, commensal bacteria engineered to express anti-malarial effector molecules. Although both approaches restrict parasite development in the mosquito, it is not

37 known how their effectiveness compares. Here we provide an in-depth assessment of transgenesis and paratransgenesis and evaluate the combination of the two approaches. Using the Q-system to drive gene expression, we engineered mosquitoes to produce and secrete two effectors - scorpine and the MP2 peptide - into the mosquito gut and salivary glands. We also engineered Serratia, a commensal bacterium capable to spread through mosquito populations, to secrete the same two effectors into the mosquito gut. Whereas both mosquito-based and bacteria-

43 based approaches strongly reduced the oocyst and sporozoite intensity, a substantially stronger

44 reduction of $P$. falciparum development was achieved when transgenesis and paratransgenesis

45 were combined. Most importantly, transmission of $P$. berghei from infected to naïve mice was maximally inhibited by the combination of the two approaches. Combining these two strategies promise to become a powerful approach to combat malaria. 


\section{Introduction}

Over 400,000 people, mostly young African children, died of malaria in 2019 (1). Whereas world malaria incidence has declined by $27 \%$ during the first 15 years of this century, in the last four years it declined by less than $2 \%$, indicating that current interventions to control this deadly disease are waning. ${ }^{1}$ The development of innovative approaches to reduce this intolerable burden is sorely needed.

The strategy of targeting the mosquito to fight malaria is based on two premises: 1) the mosquito is an obligatory vector for parasite transmission and 2) strong bottlenecks limit parasite development in the mosquito and during transmission to the mammalian host. ${ }^{2}$ The mosquito acquires the parasite when it bites an infected individual. Of the large numbers of gametocytes $\left(\sim 10^{4}\right)$ ingested by the mosquito, only a few (single digits) ookinetes succeed in traversing the mosquito gut and differentiate into oocysts, defining the first strong bottleneck. ${ }^{3}$ Each oocyst produces thousands of sporozoites, a good proportion of which invade the salivary glands, where they are stored. Only a small number of these sporozoites (on the order of $1 \%$ of total salivary gland content) are delivered when an infected mosquito bites a new individual, defining a second strong bottleneck. ${ }^{4}$

Since the early demonstration that mosquitoes can be engineered to be refractory to the parasite, ${ }^{5}$ the effectiveness of this approach has been robustly demonstrated in the laboratory by simultaneous expression of multiple effector genes (genes that stop parasite development without affecting the mosquito vector). ${ }^{6,7}$ The major current challenge is to devise means to introduce the genes that confer refractoriness into mosquito populations. This will most likely be achieved by use of CRISPR/Cas9 gene drives. ${ }^{8,9}$ In addition to technical aspects, topics to be resolved include regulatory and ethical issues related to the release of genetically modified organisms in nature.

An independent approach to suppress the mosquito vectorial capacity is to express effector genes from symbiotic bacteria rather than from the mosquito itself, an approach referred to as paratransgenesis. Paratransgenesis has the advantage that the bacteria occur in the mosquito gut in large numbers, in close proximity to the most vulnerable parasite forms. Since the early demonstration of the effectiveness of paratransgenesis to contain the spread of Trypanosoma cruzi, the causative agent of Chagas disease, by the Rhodnius prolixus vector, ${ }^{10}$ this approach has 
been developed for suppressing the mosquito's ability to vector the malaria parasite.$^{6,11,12,13}$ As

80 is the case for gene drive, the mosquito symbiont Serratia AS1 can spread through mosquito populations and be engineered to secrete effector proteins. ${ }^{14}$

This work addresses two unanswered questions: 1) which of the two genetic approaches transgenesis and paratransgenesis - is the most effective? and 2) can the two approaches be combined to enhance the effectiveness of the intervention? We use transgenic mosquitoes engineered to express effector genes in the midgut and/or salivary glands and Serratia bacteria engineered to express the same effector genes. We measured the ability of these two strategies,

\section{Results}

\section{Generation of $\boldsymbol{A n}$. stephensi mosquitoes expressing anti-malaria effectors}

To constitutively and robustly express anti-malaria effector proteins in the midgut and salivary glands of An. stephensi mosquitoes, we used the QF-QUAS binary expression system previously adapted for expression in An. gambiae. ${ }^{15,16}$ We constructed two "driver" mosquito lines that express the QF transcription factor, one driven by the constitutive salivary glandspecific (AAPP) promoter ${ }^{17}$ and the other driven by the constitutive midgut-specific peritrophin 1 (Aper1) promoter ${ }^{18,19}$ (Figure 1A). We also constructed a third "effector" mosquito line that encodes two parasite inhibiting factors (MP2 and scorpine) downstream of the QUAS promoter and driven by the QF transcription factor (Figure 1A). Crossing this effector line with either, or both, driver lines, leads to the salivary gland and/or midgut expression of parasite-inhibiting factors. The MP2 (midgut peptide 2) dodecapeptide, identified from a phage display screen, binds tightly to the mosquito midgut epithelium and inhibits $P$. falciparum invasion with high efficiency $^{20}$, whereas the scorpion (Pandinus imperator) peptide scorpine lyses malaria parasites without affecting mosquito fitness ${ }^{21,22}$. Each of the three constructs also expresses YFP (yellow eyes, salivary gland QF driver), dsRed (red eyes, midgut QF driver), or CFP (blue eyes, QUAS effector) fluorescent selection markers (Figure 1A). 
110 fluorescence microscopy (Figure 1B), and plasmid insertion was verified by PCR (Figure S1).

111 The position of genome integration was determined for each parental line by splinkerette $\mathrm{PCR}^{23}$

112 and sequencing (Table. S1). All the parental lines, except for Sg2, have insertions in intergenic

113 regions. Two of the three $\mathrm{Sg} 2$ insertions are in intergenic regions, and one in the open reading

114 frame of the gamma-glutamyltranspeptidase gene (ASTE010947). Each transgenic line was

115 propagated for over 10 generations, discarding at each generation mosquitoes not displaying the

116 correct combination of fluorescent eyes, and this resulted in homozygous lines (Table S2).

117

118

119

120

121

122

123

124

125

126

127

128

129

130

131

132

133

134

135

136

137

138

139

140

\section{Quantification of effector mRNA and protein expression}

Using reverse transcription quantitative polymerase chain reaction (RT-qPCR) we compared abundance of the endogenous mosquito Aper and AAPP transcripts with the abundance of effector transcripts originating from the same promoters but driven by the Qsystem. Transcripts derived from the Q-system were substantially higher. In the midgut, the scorpine transcript was between 44- $(P<0.01)$ and 56-fold $(P<0.01)$ higher than that of the endogenous Aper mRNA and the MP2 transcript abundance was between $49-(P<0.001)$ and 36fold $(P<0.01)$ higher, depending on the transgenic line (Figure $1 \mathrm{C}$; Table $\mathrm{S} 3$ ). In the salivary glands, scorpine transcript abundance varied between $27-(P<0.05)$ and 63-fold $(P<0.01)$ higher and MP2 transcript between 49- $(P<0.001)$ and 140-fold $(P<0.01)$ higher than that of the endogenous AAPP mRNA, depending on the transgenic line (Figure 1D; Table S4). Moreover, in the absence of a driver, transgene expression in 'E' effector mosquitoes (see Figure 1A) was undetectable (Tables S3 and S4). These results attest to the high effectiveness of the Q-system in enhancing tissue-specific transgene expression. Western blot analysis using anti-MP2 and antiscorpine antibodies confirmed the tissue specific expression of the MP2 $(6.17 \mathrm{kDa})$ and scorpine $(8.46 \mathrm{kDa})$ proteins (Figure $1 \mathrm{E}$ and $\mathrm{F})$.

\section{Mosquito fitness is not affected by effector gene expression}

To determine whether DNA integration or anti-malaria effector expression affects mosquito fitness, we analyzed the survival of WT, parental transgenic $(\mathrm{Mg}, \mathrm{Sg}$, and E) and transgeneexpressing mosquitoes. No significant longevity differences were detected for any female (Figure S2A) or male (Figure S2B) transgenic mosquitoes, compared to WT.

Next, we determined the fecundity (number of laid eggs) and fertility (percentage of hatched 
141 eggs) of WT, parental, and anti-malaria transgenic lines. Mosquitoes from all parental and anti-

142 malaria-expressing transgenic lines showed no difference in fecundity when compared to WT

143 mosquitoes (Figure S2C). As for fertility, no significant differences were detected for the Mg and

$144 \mathrm{Sg} / \mathrm{E}$ lines when compared to WT, while only marginal differences were detected for the $\mathrm{Sg}, \mathrm{E}$,

$145 \mathrm{Mg} / \mathrm{E}$ and $\mathrm{Mg} / \mathrm{Sg} / \mathrm{E}$ lines $(2.0 \%, 3.1 \%, 2.0 \%$ and $2.0 \%$ reduction, respectively) (Figure S2D).

146 To determine if transgenesis or anti-malaria gene expression in the midgut and/or in the salivary

147 glands affects blood feeding, we quantified the proportion of mosquitoes that take a blood meal

148 (feeding rate) and the amount of blood ingested per mosquito. We found no significant

149 differences (Figure S2E and S2F), suggesting that neither transgenesis nor anti-malaria gene

150 expression affects blood ingestion.

151 In summary, our data show that transgenesis and anti-malaria gene expression in the midgut

152 and/or salivary glands does not impair mosquito survival, fecundity, fertility (only minor

153 differences), and blood feeding under laboratory conditions.

\section{Effector-expressing Serratia populate the mosquito reproductive organs and are} transmitted vertically and horizontally

Previously we reported that fluorescently labelled Serratia AS1 can spread through mosquito populations and that the bacteria are inherited through multiple mosquito generations $\underline{14}$. Here we tested whether a Serratia AS1 strain (termed Serratia AS1-poly - for poly-effector) that

160 produces and secretes the same effector proteins (MP2 \& scorpine, among others) as those

161 produced by the transgenic mosquitoes can also populate mosquitoes and be transmitted from

162 one mosquito generation to another. We fed WT and $\mathrm{Mg} / \mathrm{Sg} / \mathrm{E}$ transgenic female mosquitoes

163 with Serratia AS1-poly bacteria and quantified their capacity to colonize different mosquito

164 organs and to be transmitted along consecutive mosquito generations. We found that Serratia

165 AS1-poly equally populate WT and transgenic mosquito midguts, ovaries and accessory glands

166 and are transmitted for at least three generations (Figure 2A-D). Moreover, we found that WT

167 and transgenic male mosquitoes colonized with Serratia AS1-poly transferred the bacteria

168 horizontally (sexually) to virgin WT and transgenic female mosquitoes (Figure 2E). Horizontal

169 transfer did not take place when male mosquitoes were placed with mated females, showing that

170 transfer occurs during copulation (Table S5; female mosquitoes mate only once in their 
171 lifetimes). These results suggest that recombinant Serratia AS1 can effectively populate transgenic mosquitoes and be transmitted through multiple generations.

A concern is the possibility of Serratia carried by the mosquito being incorporated into the mosquito salivary glands and being delivered with the bite of a mammalian host. To address this concern, mosquitoes previously fed with fluorescently labeled Serratia were allowed to feed on blood using a membrane feeder. The remaining blood in the feeder was collected, grown overnight in LB medium and plated. No bacteria were detected (Figure S3), even though the presence of even a single bacterium in the blood from the feeder would have been easily detected.

\section{Transgenic and paratransgenic expression of MP2 and scorpine inhibit Plasmodium}

\section{development in the mosquito}

Wild type or transgenic mosquitoes, carrying or not wild type or recombinant bacteria, were fed with the same $P$. falciparum infectious blood. Infections were followed by measuring the formation of midgut oocysts (Figure 3A) and salivary gland sporozoite numbers (Figure 3B). Expression of effector molecules in the midgut or in the salivary glands of transgenic mosquitoes significantly reduced parasite burden, whereas concomitant effector expression in both organs reduced burden to the greatest extent (81\% and $85 \%$ inhibition of mean oocyst and sporozoite numbers, respectively). Effector-expressing recombinant bacteria also significantly reduced parasite burden in WT mosquitoes (70\% and 65\% inhibition of oocyst and sporozoite numbers, respectively). Importantly, combining mosquito transgenesis with paratransgenesis led to the strongest inhibition of parasite development. Oocyst prevalence was reduced from $98 \%$ to $49 \%$ for transgenic-only mosquitoes and to $48 \%$ when transgenesis and paratransgenesis were combined. Sporozoite prevalence was reduced from $97 \%$ to $42 \%$ for transgenic-only mosquitoes and to $24 \%$ when transgenesis and paratransgenesis were combined. These results suggest that the ability of [transgenic + paratransgenic] mosquitoes to transmit the parasite may be strongly impaired, a hypothesis that was tested next.

\section{Malaria transmission is maximally impaired by combining transgenesis and} paratransgenesis 
To investigate the ability of mosquitoes to transmit the parasite from an infected to a naïve animal, we challenged naïve mice with the bite of mosquitoes that had ingested the same infectious blood meal. Four mosquito groups were investigated: 1) wild type mosquitoes (WT), 2) WT mosquitoes carrying Serratia AS1-poly (paratransgenic), 3) transgenic mosquitoes that express effectors in the midgut and salivary glands (transgenic) and 4) transgenic mosquitoes carrying Serratia AS1-poly (paratransgenic + transgenic) (Figure 4A). All four mosquito groups ingested the same number of parasites, as they were fed on the same P. berghei-infected mouse. At 21-23 days post-feeding, after the mosquito salivary glands were populated by sporozoites, either three (Figure 4B) or five (Figure 4D) mosquitoes were randomly selected and allowed to bite naïve mice. For each experiment, salivary gland sporozoite numbers were determined (Figure 4C and 4E).

When mice were challenged with the bite of three WT mosquitoes (three independent experiments with five mice each), $100 \%$ became infected (half-infection time $=5.5 \pm 0.5 \mathrm{~d}$ ) (Figure 4B) and their salivary glands had a median 8,400 sporozoites (Figure 4C). With mosquitoes carrying Serratia AS1-poly (paratransgenic), 26.7\% of the mice were protected (half-infection time $7.1 \pm 0.7 \mathrm{~d}$ ), and their salivary glands had a median of 2,100 sporozoites (74\% lower than WT mosquitoes). With transgenic mosquitoes, 67\% of the mice were protected, and their salivary glands had a median of 900 sporozoites ( $92 \%$ lower than WT mosquitoes). With [paratransgenic + transgenic] mosquitoes, 93\% mice were protected, and their salivary glands had a median of zero sporozoites (100\% lower than WT mosquitoes).

When mice were challenged with the bite of five WT mosquitoes (Figure 4D and 4E), only one mouse out of $15(6.7 \%)$ did not get infected (half-infection time $=5.6 \pm 0.7 \mathrm{~d}$ ).

Paratransgenesis, protected $26.7 \%$ mice (half-infection time $=8.3 \pm 1.0$ ), transgenesis protected $47 \%$ mice (half-infection time $=10.1 \pm 1.0 \mathrm{~d}$ ) and [paratransgenesis + transgenesis] protected $80 \%$ mice. The salivary gland sporozoite number (Figure $4 \mathrm{E}$ ) was similar to that observed for experiments with three mosquito bites (Figure 4C).

In summary, our data shows that transgenic and paratransgenic expression of MP2 and scorpine are both effective in impairing transmission, and that the combination of the two complementary strategies is considerably more effective. An even higher protection is expected from the bite of one infected mosquito, which is the most likely scenario in the field. 


\section{Discussion}

In this study, we report the development of transgenic mosquitoes and paratransgenic bacteria expressing anti-plasmodial effector molecules and the effect of these two technologies, individually or in combination, on the transmission of Plasmodium parasites. The Q-binary expression system was used to express effector genes in the mosquito midgut and salivary glands. Notably, effector mRNA abundance was about 50-times higher than that of the endogenous genes, consistent with the high effectiveness of the Q-system in Drosophila. ${ }^{15}$ Some QF toxicity was reported when the Q-system was first used in Drosophila. ${ }^{15,16}$ Of note, expression of neither the QF transcription factor nor the anti-malaria effectors affected mosquito longevity, blood meal uptake or offspring production under laboratory conditions. Additional experiments are required to test the fitness of these transgenic mosquitoes under field conditions.

We selected two potent effectors molecules, MP2 and scorpine, to block the development of Plasmodium in the mosquito. MP2 is a 12-amino-acid peptide that likely targets a midgut receptor for ookinete traversal, ${ }^{20}$ and scorpine is an antimicrobial toxin hybrid between a cecropin and a defensin, that lyses Plasmodium ookinetes. ${ }^{21}$ Scorpine expressed by the entomopathogenic fungus Metarhizium in the mosquito hemocoel strongly inhibits ( $\sim 90 \%)$ salivary gland sporozoite numbers. ${ }^{24}$ Transgenic mosquitoes expressing this effector in the salivary glands were also highly effective in reducing sporozoite numbers (this work). Furthermore, expression of both effector genes in the midgut and the salivary glands led to a much stronger decrease of salivary gland sporozoite numbers than the expression of the effectors in either of these organs alone. A number of effectors have already been individually tested in paratransgenesis experiments. ${ }^{14}$ Going forward, the combination of different effectors and the use of mosquitoes and bacteria expressing different effector sets should be explored, to achieve maximum blocking activity.

That expression of anti-malaria effectors in the salivary glands inhibited oocyst development in the midgut is most likely explained by the fact that mosquitoes ingest saliva with the blood meal, in this way incorporating effector proteins into the blood bolus. ${ }^{25}$ Effector molecules secreted in the saliva could conceivable be injected into the dermis of the host during blood feeding. Scorpine has been shown to be non-toxic to insect cells, ${ }^{26}$ whereas MP2 toxicity has not been determined. However, their toxicity has not been tested in the presence of mosquito saliva. Therefore, further studies are needed to determine if the delivery of these molecules by 
the mosquito bite could induce physiological responses.

Experiments seeking evidence for possible bacteria transmission with a mosquito bite yielded negative results (Figure S3), suggesting that mosquitoes cannot inoculate the bacteria while feeding on a host. It was previously shown that secretion of effector proteins by recombinant Pantoea, ${ }^{6}$ Serratia $^{14}$ or Asaia $^{12}$ bacteria into the midgut inhibits Plasmodium development and that Serratia AS1 is transmitted from one mosquito generation to the next. ${ }^{14}$ What was not known is whether engineering Serratia to produce and secrete large amounts of proteins would affect their fitness and ability to be transmitted. Our experiments showed that the engineered Serratia were efficiently transmitted from one mosquito generation to the next, a result that bodes well for the implementation of the paratransgenesis strategy in the field.

This project was based on two basic premises: (i) transgenesis and paratransgenesis are not mutually exclusive and (ii) both strategies result in impairment of parasite development in the mosquito. As such, our experiments addressed the question of whether a combination of the two strategies would result in enhanced transmission-blocking effectiveness. The combination of transgenesis and paratransgenesis greatly reduced parasite development in the mosquito and most importantly, it resulted in a high-level reduction of transmission from an infected to a naïve mouse, compared to individual interventions. When mice were bitten by three [transgenic + paratransgenic] mosquitoes, $93 \%$ of the mice were protected from infection as compared with zero protection when mice were bitten by WT mosquitoes that acquired the parasites from the same infected mouse. In the field, where the density of infected mosquitoes is low even in hightransmission areas, it is unlikely that people will be consecutively bitten by more than one infected mosquito, and protection from transmission is expected to be very high. For translating these findings to the field, the testing of different combinations of effectors, both for transgenesis and paratransgenesis, may further improve the effectiveness of the approach.

Whereas both transgenesis and paratransgenesis have been shown to be highly effective in a lab setting, the challenge is to implement this new containment strategies in the field. In addition to address regulatory and ethical issues connected with the release of recombinant organisms in nature, a major technical issue to be solved is how to introduce the blocking transgenes into mosquito populations in the field. In this respect, CRISPR/Cas9 technology has afforded the development of promising gene drive systems ${ }^{27,28,29}$ focused on population suppression or population modification strategies. Population reduction leaves an empty 
294 biological niche that upon cessation of reduction pressure, will result in recolonization by the 295 same or other mosquito species. In contrast, population modification results in a more stable 296 state, with a biological niche occupied by mosquitoes that are poor transmitters. Similarly, 297 efficient spread of recombinant bacteria into mosquito populations has been demonstrated in a 298 laboratory setting (, ${ }^{14}$ this work), indicating a promising path toward the field implementation of 299 the most efficient [transgenesis + paratransgenesis] strategy. The recent finding that a naturally 300 occurring and non-modified Serratia can spread through mosquito populations while strongly 301 suppressing Plasmodium development, ${ }^{30}$ significantly increases the feasibility of moving 302 paratransgenesis into the field, as it bypasses concerns relating to the release of genetically 303 modified organisms in nature. In the field, we envision the use of attractive sugar feeding stations 304 for Serratia introduction into mosquito populations. ${ }^{31}$ Female mosquitoes that acquire the 305 bacteria will seed the breeding sites when they lay eggs. ${ }^{14}$ Notably, transgenesis and 306 paratransgenesis are not envisioned to be implemented by themselves. Both are compatible with 307 current vector and malaria control measures such as insecticide-based mosquito control, mass 308 drug administration, and vaccines, and their added implementation promises to substantially 309 enhance the effectiveness of intervention of disease transmission.

310 In summary, we show that the Q-binary system to express anti-Plasmodium effectors in 311 the mosquito is highly efficient. We also show that in addition to inhibiting parasite development, 312 recombinant Serratia AS1 is horizontally and vertically transmitted across multiple mosquito 313 generations, which is a bacteria counterpart of gene drive. A major conclusion of this work is that 314 the combination of transgenesis with paratransgenesis provides maximum parasite blocking 315 activity and has high potential for fighting malaria. 


\section{Material and Methods}

\section{Animal Handling and Ethics Protocol}

Ethics statement

This study was carried out in accordance with the guidelines of the Johns Hopkins

\section{Mosquitoes rearing and parasite culture}

Anopheles stephensi Nijmegen $\operatorname{strain}^{32}$ and An. stephensi transgenic lines were reared as previously described $\frac{33}{}$. For fitness evaluation, the mosquitoes were fed on Swiss Webster mice.

Female An. stephensi were infected with P. falciparum gametocyte cultures via membrane feeding. P. falciparum NF54 gametocytes were produced according to Tripathi et al., . Briefly, the parasites were maintained in O+ human erythrocytes using RPMI 1640 medium supplemented with $25 \mathrm{mM}$ HEPES, $50 \mathrm{mg} / \mathrm{L}$ hypoxanthine, $25 \mathrm{mM} \mathrm{NaHCO}$, and 10\% (v/v) heat-inactivated type $\mathrm{O}+$ human serum (Interstate Blood Bank, Inc.) at $37{ }^{\circ} \mathrm{C}$ and with a gas mixture of $5 \% \mathrm{O}_{2}, 5 \% \mathrm{CO}_{2}$, and balanced $\mathrm{N}_{2}$. For feeding, 14-17-day-old mature gametocytes were pelleted by centrifugation ( $5 \mathrm{~min}, 2,500 \mathrm{~g}$ ), resuspended with $\mathrm{O}+$ human $\mathrm{RBC}$ to $0.15 \%$ $0.2 \%$ gametocytemia and diluted to $40 \%$ hematocrit with human serum. All manipulations were

Plasmid constructs

The pXL-BACIIECFP-15XQUAS-TATA-MP2-SV40-15XQUAS-TATA-scorpine-SV40 containing the MP2 and Scorpine expression cassette and the ECFP gene under the eye-specific promoter 3xP3 was used to generate the parental QUAS-[MP2+scorpine] effector lines (Table

342 (Figure S4). The sequence was amplified using primers MP2-ScopineF and MP2-ScopineR

343 (Table S7), and In-Fusion-cloned into plasmid pXL-BACIIECFP-15XQUAS-TATA-SV40 ${ }^{15}$

344 previously linearized with XhoI.

The pXL-BACII-DsRed-AsAper-QF2-hsp70 containing the QF2 transcription factor under the control of the midgut specific AsAper promoter and the DsRed marker driven by the

347 eye-specific promoter 3xP3 was used to generate the parental Mg-QF driver line. The AsAper 
promoter (1.5 kb) (Figure S4) was PCR amplified from An. stephensi gDNA with primers MgPF and MgPR (Table S7). The PCR product was In-Fusion-cloned into plasmid pXL-BACIIDsRed-QF2-hsp70 ${ }^{15}$ previously linearized with XhoI.

The pXL-BACII-YFP-AsAAPP-QF2-hsp70 containing the QF2 transcription factor under the control of the midgut specific AsAAP promoter and the YFP marker driven by the eyespecific promoter 3xP3, was used to generate the parental Sg-QF driver lines. The YFP coding sequence was amplified using primers YFPF and YFPR (Table. S7) (Figure S4). The PCR product was In-Fusion-cloned into plasmid pXL-BACII-DsRed-QF2-hsp70 previously digested with ApaI and NotI to produce plasmid pXL-BACII-YFP-QF2-hsp70. The AsAAPP promoter consisting of a $1.7 \mathrm{~kb}$ upstream of the start $\operatorname{codon}^{19}$ was PCR-amplified from An. stephensi gDNA using primers SgPF and SgPR (Table. S7) (Fig. S4). The PCR product was In-Fusioncloned into plasmid pXL-BACII-YFP-QF2-hsp70 previously linearized with XhoI.

\section{Generation of transgenic mosquitoes}

The plasmid constructs were microinjected into An. stephensi embryos as described. ${ }^{35}$ Briefly, transformation plasmids were purified using the EndoFree Maxi Prep Kit (Qiagen) and resuspended in injection buffer $(0.1 \mathrm{mM} \mathrm{NaHPO} 4 \mathrm{pH} 6.8$ and $5 \mathrm{mM} \mathrm{KCl})$ at a concentration of $250 \mathrm{ng} / \mu \mathrm{l}$ for the transformation plasmid and $200 \mathrm{ng} / \mu \mathrm{l}$ for the helper plasmid encoding the transposase. The plasmid mix was injected into An. stephensi embryos using a FemtoJet Microinjector (Eppendorf). Third instar larvae of $\mathrm{G}_{0}$ survivors were screened for transient expression of the 3xP3-dsRed marker (red eyes), 3xP3-YFP marker (yellow eyes), 3xP3-CFP marker (blue eyes). Adults obtained from the fluorescent marker screening were crossed to WT mosquitoes to generate independent transgenic lines. The data for these injections are summarized in Table S8.

For each of the parental transgenic lines, splinkerette $\mathrm{PCR}^{23}$ and PCR sequencing were used to determine the transgene insertion site into the An. stephensi genome. (Two rounds of amplifications were conducted with 1X Phusion High-Fidelity PCR Master Mix with HF Buffer (Thermo Fisher Scientific). The primers used are shown in Table S7. The amplified PCR products were resolved in a 1.5\% agarose gel stained with ethidium bromide, and the amplified DNA bands from the 5' and 3' ends were individually excised and purified with QIAquick ${ }^{\circledR}$ Gel 
Extraction Kit (QIAGEN). Purified PCR products were cloned into pJET1.2/blunt plasmid (Thermo Fisher Scientific) and transformed into NEB 5-alpha Competent Escherichia coli (High Efficiency, Thermo Fisher Scientific). Plasmids were isolated from individual colonies and sequenced with the universal primers pJET12F and pJET12R (Eurofins). The sequences were aligned to the An. stephensi genome using VectorBase and NCBI BLAST to identify the location of transgene insertion sites (Figure S1).

To obtain homozygous lines, each transgenic line was propagated for more than 10 generations, discarding at each generation mosquito larvae not displaying the expected fluorescent eyes. To verify homozygosity of the transgenic lines, 10 females of each line were mated with 10 WT male mosquitoes, fed blood, eggs were collected and reared to larvae. The larvae were individually inspected for expression of the fluorescent protein marker(s). Absence of the expected fluorescence would indicate that the parent female was heterozygous for this dominant marker.

To induce midgut- or salivary gland-specific expression of MP2 and scorpine, QF driver lines were crossed to QUAS-[MP2+scorpine] effector lines. The offspring of each cross was selected by the specific combination of eye fluorescence reporters (Figure 1B).

\section{Quantitative reverse transcription polymerase chain reaction (qRT-PCR)}

Tissue specific expression of MP2 and scorpine mRNAs in An. stephensi transgenic lines was evaluated by RT-PCR. Salivary glands and midguts were dissected from female mosquitoes in ice-cold $200 \mu 1$ TRIzol® (Thermo Fisher Scientific). Total RNA was extracted according to TRIzol ${ }^{\circledR}$ manufacturer's protocol, resuspended in RNAse free water, and treated with RQ1 RNase-Free DNase ${ }^{\circledR}$ (Promega; Madison, WI, USA). After RNA quantification using a DeNovix DS-11 spectrophotometer, first-strand cDNA was synthesized for each sample using Superscript III (Invitrogen) with random hexamers (Invitrogen) and $500 \mathrm{ng}$ of total RNA per sample. cDNA was treated with RNase H (New England Biolabs) for 10 min at $37{ }^{\circ} \mathrm{C}$ and stored at $-70{ }^{\circ} \mathrm{C}$ until use. The cDNA was used as template in PCR reactions containing the Taq $2 \mathrm{X}$ Master Mix (New England Biolabs) and $5 \mu \mathrm{M}$ of MP2- and scorpine-specific primers (Table S7). Amplification of S7 ribosomal mRNA was used as reference. ${ }^{36}$ PCR conditions were: 1 hot start at $95{ }^{\circ} \mathrm{C}$ for $30 \mathrm{sec} ; 35 \mathrm{cycles}$ of denaturation at $95{ }^{\circ} \mathrm{C}$ for $30 \mathrm{sec}$, annealing at $56{ }^{\circ} \mathrm{C}$ for $30 \mathrm{sec}$, and elongation at $68{ }^{\circ} \mathrm{C}$ for $30 \mathrm{sec}$; followed by a final extension at $68{ }^{\circ} \mathrm{C}$ for $5 \mathrm{~min}$; and $4{ }^{\circ} \mathrm{C}$ 
indefinitely.

\section{Mice immunization}

Scorpine epitope (CEKHCQTSGEKGYCHGT, the N-terminus was conjugated to KLH) and MP2 epitope (ACYIKTLHPPCS, the N-terminus was conjugated to KLH) were synthesized by Peptide 2.0 Inc. About 6-8-week-old C57BL/6 mice were immunized with $20 \mu \mathrm{g}(50 \mu \mathrm{l})$ purified antigen in PBS using Addavax (Invivogen, San Diego, CA) as the adjuvant. A total of 50 $\mu l$ adjuvant was mixed with $50 \mu l$ antigen, and the mixture was administered intramuscularly in both anterior tibialis muscles (50 $\mu \mathrm{l}$ per leg). Mice were immunized twice at two-week intervals. Serum was collected 14-21 days after administration of the last booster. ${ }^{37}$

\section{Commercial antibodies}

Rabbit anti- $\alpha$-tubulin was purchased from Sigma (cat\# SAB3501072) and goat anti-rabbit IgG HRP-conjugated and goat anti-mouse IgG HRP-conjugated were purchased from Cell

\section{Signaling (cat\# 7076S).}

\section{Western blotting}

MP2 and scorpine protein synthesis in midgut and salivary glands of the transgenic lines was evaluated by Western blot. Five midguts and ten salivary glands were dissected in PBS and placed in microtubes containing RIPA Buffer ${ }^{\circledR}$ (Thermo Fisher Scientific), 1\% Halt ${ }^{\mathrm{TM}}$ Protease Inhibitor Cocktail (Thermo Fisher Scientific), and 0.1 mM PMSF (Sigma-Aldrich). Samples were homogenized and stored at $-70^{\circ} \mathrm{C}$. An equivalent of 0.25 midgut and 5 salivary glands were resolved in a NuPAGE ${ }^{\mathrm{TM}}$ 10\% Bis-Tris Protein Gel (Invitrogen) under reducing conditions and transferred to a PVDF membrane Invitrogen ${ }^{\mathrm{TM}}$ Power Blotter Select Transfer Stacks. After the transfer, the membrane was washed with TBST 1\% (Sigma-Aldrich), incubated with blocking buffer $(5 \%$ milk powder in TBST $1 \%)$ overnight at $4{ }^{\circ} \mathrm{C}$, and probed with mouse antiMP2 or anti-scorpine at a 1:1,000 dilution in TBST $1 \%$ overnight at $4{ }^{\circ} \mathrm{C}$. The membrane was washed and incubated with an anti-mouse HRP-linked antibody (Cell Signaling) at a 1:10,000 dilution in TBST $1 \%$ for $2 \mathrm{~h}$ at room temperature. Detection was done with the SuperSignal ${ }^{\mathrm{TM}}$ West Dura Extended Duration Substrate Chemiluminescent Substrate (Thermo Fisher Scientific), and imaged using an Azure Imager c600 ${ }^{\circledR}$ (Azure Biosystems). 


\section{Mosquito survival, fecundity, and fertility}

To measure mosquito survival, two-day-old adult male and female mosquitoes $(\mathrm{n}=100)$ were separately placed in a cage with cotton pads soaked in $10 \%$ sucrose solution and kept in the insectary. Female mosquitoes were allowed to blood feed on an anesthetized mouse for 30 min and allowed to lay eggs. Mortality of female and male mosquitoes was monitored 3 times per week. The differences among the survival curves (three independent replicates) were analyzed with the Log-rank (Mantel-Cox) test, using the WT as controls.

To assess fecundity (number of laid eggs) and fertility (percentage of hatched eggs), twoday-old adult females were blood-fed on anesthetized mice for $30 \mathrm{~min}$. Only fully engorged females were used for these experiments. Two days after blood-feeding, 20 females were individually placed in $50 \mathrm{ml}$ tubes containing a small cup with filter paper soaked in $2 \mathrm{ml}$ of distilled water as a oviposition substrate. After three days, the filter papers with eggs were removed, and the number of eggs per mosquito was counted using a dissecting microscope. After counting, the eggs were placed in paper cups with $50 \mathrm{ml}$ of distilled water to allow hatching. fertility was determined as the number of larvae divided by the total number of eggs. Fecundity and fertility of the transgenic lines were compared to WT mosquitoes, and all the experiments were repeated for a total of three biological replicates. meal. ${ }^{38}$ Transgenic and WT mosquitoes were fed with a 1:1 mixture of plasma and RBCs

464 (Interstate Blood Bank Inc.) using membrane feeders. After feeding, the midguts of ten fully engorged females were dissected and homogenized individually in $1 \mathrm{ml}$ of distilled water. Unfed mosquitoes were used as the negative control. Protein-bound heme $(410 \mathrm{~nm})$ was measured for each individual midgut with a Versa max microscope Reader and recorded with Softmax pro 5.3. Readings were compared among the groups using Student's t test.

\section{Bacteria administration to An. stephensi mosquitoes}

471 After culturing at $28^{\circ} \mathrm{C}$ overnight, bacteria were washed with sterile PBS and resuspended to a 
472 final concentration of $10^{9} / \mathrm{ml}$. After a $3 \mathrm{~h}$ starvation, mosquitoes were fed overnight on $10^{7} \mathrm{CFU}$

473 bacteria ( $A S 1$-poly, apramycin resistance) per $\mathrm{ml}$ of 5\% sugar. Mosquitoes were surface-

474 sterilized with cold $75 \%$ ethanol for $3 \mathrm{~min}$ and washed three times with sterile PBS. Midguts

475 were dissected under sterile conditions at different time points before and after a blood meal and

476 homogenized in sterile PBS. Bacterial number was determined by plating ten-fold serial dilutions

477 of the homogenates on LB agar plates containing $50 \mu \mathrm{g} / \mathrm{ml}$ apramycin and ampicillin (bacteria

478 from non-infected mosquitoes cannot grow on LB agar plates containing $50 \mu \mathrm{g} / \mathrm{ml}$ apramycin

479 and ampicillin) and incubating at $28^{\circ} \mathrm{C}$ for $24 \mathrm{~h}$.

\section{Effect of bacteria on mosquito infection by Plasmodium falciparum}

Serratia bacteria were administered overnight to female An. stephensi with a cotton pad

soaked with a $5 \%$ sucrose solution containing $10^{7}$ bacteria $/ \mathrm{ml}$ or no bacteria, and $2 \mathrm{~d}$ later, mosquitoes were kept at $27{ }^{\circ} \mathrm{C}$ and $80 \%$ relative humidity. Midguts were dissected in $1 \times \mathrm{PBS}$ at waiting for at least $5 \mathrm{~min}$ to allow sporozoites to sediment to the bottom of the chamber. Sporozoites were counted using a Leica phase-contrast microscope. Parasite numbers among control and experimental groups were compared using the nonparametric Mann-Whitney test (GraphPad, Prism).

\section{Effect of bacteria on mosquito infection by Plasmodium berghei}

Bacteria were cultured overnight in LB medium and washed three times with sterile PBS. Two-day-old mosquitoes were fed overnight on a cotton pad soaked with a $5 \%$ sucrose solution containing or not $10^{7}$ bacteria/ml. Two days later, mosquitoes were fed on a $P$. berghei-infected mouse (1-2\% of parasitemia and 1 exflagellation per 10 fields). Unfed mosquitoes were removed, and fully engorged mosquitoes were provided with 5\% (wt/vol) sterile sucrose solution

501 and maintained at $19{ }^{\circ} \mathrm{C}$ and $80 \%$ relative humidity. Midguts were dissected on day 12 after the

502 blood meal, stained with $0.1 \%$ (wt/vol) mercurochrome for determining oocyst load. Salivary 
glands were dissected at $21 \mathrm{~d}$ post-infection for sporozoite determination. Transgenic and WT mosquitoes were simultaneously fed on the same $P$. berghei-infected mouse to assure that control and experimental mosquitoes ingested the same number of parasites.

\section{Serratia vertical, venereal and transstadial transmission}

To test vertical transmission, $A S 1$-poly were introduced into two-day-old adult female mosquitoes by feeding them overnight on a cotton pad moistened with $5 \%$ sterile sucrose containing $10^{7}$ bacteria/ml. Two days later, mosquitoes were fed on a healthy mouse and were then allowed to lay eggs on a damp filter paper in individual oviposition tubes. Eggs were collected into a tube containing $300 \mu \mathrm{l}$ sterile $1 \times \mathrm{PBS}$ and homogenized. The bacterial load was determined by plating ten-fold serial dilutions of the egg homogenates on LB agar plates containing $50 \mu \mathrm{g} / \mathrm{ml}$ of apramycin and ampicillin and incubating the plates at $28{ }^{\circ} \mathrm{C}$ for $24 \mathrm{~h}$ for colony counting. Rearing of larvae to adults followed standard protocol. A total of 10 male and 10 female adults were sampled and examined by plating adult midgut homogenates on LB agar plates containing apramycin and ampicillin. To test the efficiency of Serratia transmission through multiple generations, the mosquitoes were reared without providing additional Serratia AS1 and maintained for three consecutive generations. At each generation, 10 female and male adults were sampled for examining the presence of $A S 1$-poly effectors.

For male-to-female venereal transmission tests, Serratia were introduced into newly emerged virgin male mosquitoes by feeding them overnight on a cotton pad moistened with $5 \%$ sugar solution containing $10^{7}$ bacteria/ml. Twenty Serratia-carrying males were then allowed to mate with 20 three-day-old virgin females. Three days after mating, 10 females were sampled and examined for bacteria in the female midgut, ovary and spermatheca.

\section{Transmission from infected to naïve mice}

Transgenic and WT mosquitoes were simultaneously fed on the same $P$. berghei-infected mouse and unfed or partially fed mosquitoes were removed. Midguts from a small number of mosquitoes were dissected at $12 \mathrm{~d}$ post-feeding to determine the infection status by counting oocyst numbers. At $\sim 21-23$ days post-feeding, three or five mosquitoes were randomly selected from the cage and allowed to feed on non-infected mice (challenge). Mosquitoes that did not take 
534 a blood meal were replaced until the final number of mosquitoes for each group (three or five)

535 was reached. The salivary glands of most mosquitoes were dissected for counting sporozoites. A

536 total of five mice were used per experiment and three biological replicates were conducted for a

537 total of 15 mice per mosquito group. After mosquito challenge, mice were monitored daily for 14

$538 \mathrm{~d}$ to determine blood-stage infection using Giemsa-stained blood smears. 


\section{References:}

1. World Health, O. (2020). World malaria report 2020: 20 years of global progress and challenges (World Health Organization).

2. Smith, R.C., Vega-Rodriguez, J., and Jacobs-Lorena, M. (2014). The Plasmodium bottleneck: malaria parasite losses in the mosquito vector. Mem Inst Oswaldo Cruz 109, 644-661.

3. Wang, S., and Jacobs-Lorena, M. (2013). Genetic approaches to interfere with malaria transmission by vector mosquitoes. Trends Biotechnol 31, 185-193. 10.1016/j.tibtech.2013.01.001.

4. Vanderberg, J.P. (1977). Plasmodium berghei: quantitation of sporozoites injected by mosquitoes feeding on a rodent host. Exp Parasitol 42, 169-181. 10.1016/0014-4894(77)90075-3.

5. Ito, J., Ghosh, A., Moreira, L.A., Wimmer, E.A., and Jacobs-Lorena, M. (2002). Transgenic anopheline mosquitoes impaired in transmission of a malaria parasite. Nature 417, 452-455. $10.1038 / 417452 \mathrm{a}$.

6. Wang, S., Ghosh, A.K., Bongio, N., Stebbings, K.A., Lampe, D.J., and Jacobs-Lorena, M. (2012). Fighting malaria with engineered symbiotic bacteria from vector mosquitoes. Proc Natl Acad Sci U S A 109, 12734-12739. 10.1073/pnas.1204158109.

7. Dong, Y., Simoes, M.L., and Dimopoulos, G. (2020). Versatile transgenic multistage effector-gene combinations for Plasmodium falciparum suppression in Anopheles. Sci Adv 6, eaay5898. 10.1126/sciadv.aay5898.

8. Carballar-Lejarazu, R., Ogaugwu, C., Tushar, T., Kelsey, A., Pham, T.B., Murphy, J., Schmidt, H., Lee, Y., Lanzaro, G.C., and James, A.A. (2020). Next-generation gene drive for population modification of the malaria vector mosquito, Anopheles gambiae. Proc Natl Acad Sci U S A 117, 22805-22814. 10.1073/pnas.2010214117.

9. Quinn, C.M., and Nolan, T. (2020). Nuclease-based gene drives, an innovative tool for insect vector control: advantages and challenges of the technology. Curr Opin Insect Sci 39, 77-83. 10.1016/j.cois.2020.03.007.

10. Durvasula, R.V., Gumbs, A., Panackal, A., Kruglov, O., Aksoy, S., Merrifield, R.B., Richards, F.F., and Beard, C.B. (1997). Prevention of insect-borne disease: an approach using transgenic symbiotic bacteria. Proc Natl Acad Sci U S A 94, 3274-3278. 10.1073/pnas.94.7.3274.

11. Yoshida, S., Matsuoka, H., Luo, E., Iwai, K., Arai, M., Sinden, R.E., and Ishii, A. (1999). A singlechain antibody fragment specific for the Plasmodium berghei ookinete protein Pbs 21 confers transmission blockade in the mosquito midgut. Mol Biochem Parasitol 104, 195-204. 10.1016/s01666851(99)00158-9.

12. Shane, J.L., Grogan, C.L., Cwalina, C., and Lampe, D.J. (2018). Blood meal-induced inhibition of vector-borne disease by transgenic microbiota. Nat Commun 9, 4127. 10.1038/s41467-018-06580-9.

13. Riehle, M.A., Moreira, C.K., Lampe, D., Lauzon, C., and Jacobs-Lorena, M. (2007). Using bacteria to express and display anti-Plasmodium molecules in the mosquito midgut. Int J Parasitol 37, 595-603. 10.1016/j.ijpara.2006.12.002.

14. Wang, S., Dos-Santos, A.L.A., Huang, W., Liu, K.C., Oshaghi, M.A., Wei, G., Agre, P., and JacobsLorena, M. (2017). Driving mosquito refractoriness to Plasmodium falciparum with engineered symbiotic bacteria. Science 357, 1399-1402. 10.1126/science.aan5478.

15. Potter, C.J., Tasic, B., Russler, E.V., Liang, L., and Luo, L. (2010). The Q system: a repressible binary system for transgene expression, lineage tracing, and mosaic analysis. Cell 141, 536-548. 10.1016/j.cell.2010.02.025.

16. Riabinina, O., Task, D., Marr, E., Lin, C.C., Alford, R., O'Brochta, D.A., and Potter, C.J. (2016). Organization of olfactory centres in the malaria mosquito Anopheles gambiae. Nat Commun 7 , 13010. 10.1038/ncomms13010.

17. Shen, Z., and Jacobs-Lorena, M. (1998). A type I peritrophic matrix protein from the malaria vector Anopheles gambiae binds to chitin. Cloning, expression, and characterization. J Biol Chem 273, 17665-17670. 10.1074/jbc.273.28.17665.

18. Abraham, E.G., Donnelly-Doman, M., Fujioka, H., Ghosh, A., Moreira, L., and Jacobs-Lorena, M. (2005). Driving midgut-specific expression and secretion of a foreign protein in transgenic 
mosquitoes with AgAper1 regulatory elements. Insect Mol Biol 14, 271-279. 10.1111/j.13652583.2004.00557.x.

19. Yoshida, S., and Watanabe, H. (2006). Robust salivary gland-specific transgene expression in Anopheles stephensi mosquito. Insect Mol Biol 15, 403-410. 10.1111/j.1365-2583.2006.00645.x.

20. Vega-Rodriguez, J., Ghosh, A.K., Kanzok, S.M., Dinglasan, R.R., Wang, S., Bongio, N.J., Kalume, D.E., Miura, K., Long, C.A., Pandey, A., and Jacobs-Lorena, M. (2014). Multiple pathways for Plasmodium ookinete invasion of the mosquito midgut. Proc Natl Acad Sci U S A 111, E492-500. 10.1073/pnas.1315517111.

21. Conde, R., Zamudio, F.Z., Rodriguez, M.H., and Possani, L.D. (2000). Scorpine, an anti-malaria and anti-bacterial agent purified from scorpion venom. FEBS Lett 471, 165-168. 10.1016/s00145793(00)01384-3.

22. Gao, B., Xu, J., Rodriguez Mdel, C., Lanz-Mendoza, H., Hernandez-Rivas, R., Du, W., and Zhu, S. (2010). Characterization of two linear cationic antimalarial peptides in the scorpion Mesobuthus eupeus. Biochimie 92, 350-359. 10.1016/j.biochi.2010.01.011.

23. Potter, C.J., and Luo, L. (2010). Splinkerette PCR for mapping transposable elements in Drosophila. PLoS One 5, e10168. 10.1371/journal.pone.0010168.

24. Fang, W., Vega-Rodriguez, J., Ghosh, A.K., Jacobs-Lorena, M., Kang, A., and St Leger, R.J. (2011). Development of transgenic fungi that kill human malaria parasites in mosquitoes. Science 331, 10741077. 10.1126/science.1199115.

25. Luo, E., Matsuoka, H., Yoshida, S., Iwai, K., Arai, M., and Ishii, A. (2000). Changes in salivary proteins during feeding and detection of salivary proteins in the midgut after feeding in a malaria vector mosquito, Anopheles stephensi (Diptera : Culicidae). Medical Entomology and Zoology 51, 13-20. 10.7601/mez.51.13.

26. Carballar-Lejarazu, R., Rodriguez, M.H., de la Cruz Hernandez-Hernandez, F., Ramos-Castaneda, J., Possani, L.D., Zurita-Ortega, M., Reynaud-Garza, E., Hernandez-Rivas, R., Loukeris, T., Lycett, G., and Lanz-Mendoza, H. (2008). Recombinant scorpine: a multifunctional antimicrobial peptide with activity against different pathogens. Cell Mol Life Sci 65, 3081-3092. 10.1007/s00018-008-8250-8.

27. Nolan, T. (2021). Control of malaria-transmitting mosquitoes using gene drives. Philos Trans R Soc Lond B Biol Sci 376, 20190803. 10.1098/rstb.2019.0803.

28. Simoni, A., Hammond, A.M., Beaghton, A.K., Galizi, R., Taxiarchi, C., Kyrou, K., Meacci, D., Gribble, M., Morselli, G., Burt, A., et al. (2020). A male-biased sex-distorter gene drive for the human malaria vector Anopheles gambiae. Nat Biotechnol 38, 1054-1060. 10.1038/s41587-020-05081.

29. Adolfi, A., Gantz, V.M., Jasinskiene, N., Lee, H.F., Hwang, K., Terradas, G., Bulger, E.A., Ramaiah, A., Bennett, J.B., Emerson, J.J., et al. (2020). Efficient population modification gene-drive rescue system in the malaria mosquito Anopheles stephensi. Nat Commun 11, 5553. 10.1038/s41467-02019426-0.

30. Gao, H., Bai, L., Jiang, Y., Huang, W., Wang, L., Li, S., Zhu, G., Wang, D., Huang, Z., Li, X., et al. (2021). A natural symbiotic bacterium drives mosquito refractoriness to Plasmodium infection via secretion of an antimalarial lipase. Nat Microbiol 6, 806-817. 10.1038/s41564-021-00899-8.

31. Bilgo, E., Vantaux, A., Sanon, A., Ilboudo, S., Dabire, R.K., Jacobs-Lorena, M., and Diabate, A. (2018). Field assessment of potential sugar feeding stations for disseminating bacteria in a paratransgenic approach to control malaria. Malar J 17, 367. 10.1186/s12936-018-2516-X.

32. Feldmann, A.M., and Ponnudurai, T. (1989). Selection of Anopheles stephensi for refractoriness and susceptibility to Plasmodium falciparum. Med Vet Entomol 3, 41-52. 10.1111/j.13652915.1989.tb00473.x.

33. Huang, W., Wang, S., and Jacobs-Lorena, M. (2020). Self-limiting paratransgenesis. PLoS Negl Trop Dis 14 , e0008542. 10.1371/journal.pntd.0008542.

34. Tripathi, A.K., Mlambo, G., Kanatani, S., Sinnis, P., and Dimopoulos, G. (2020). Plasmodium falciparum Gametocyte Culture and Mosquito Infection Through Artificial Membrane Feeding. J Vis Exp. 10.3791/61426. 
35. Volohonsky, G., Terenzi, O., Soichot, J., Naujoks, D.A., Nolan, T., Windbichler, N., Kapps, D., Smidler, A.L., Vittu, A., Costa, G., et al. (2015). Tools for Anopheles gambiae Transgenesis. G3 (Bethesda) 5, 1151-1163. 10.1534/g3.115.016808.

36. Zhang, J., Huang, F.S., Xu, W.Y., Wang, Y., Zhou, T.L., and Duan, J.H. (2011). Plasmodium yoelii: correlation of TEP1 with mosquito melanization induced by nitroquine. Exp Parasitol 127, 52-57. 10.1016/j.exppara.2010.06.032.

37. Cha, S.J., McLean, K.J., and Jacobs-Lorena, M. (2018). Identification of Plasmodium GAPDH epitopes for generation of antibodies that inhibit malaria infection. Life Sci Alliance 1, e201800111. 10.26508/lsa.201800111.

Alves, E.S.T.L., Radtke, A., Balaban, A., Pascini, T.V., Pala, Z.R., Roth, A., Alvarenga, P.H., Jeong, Y.J., Olivas, J., Ghosh, A.K., et al. (2021). The fibrinolytic system enables the onset of Plasmodium infection in the mosquito vector and the mammalian host. Sci Adv 7. 10.1126/sciadv.abe3362.

\section{Acknowledgments}

We thank the Insectary and Parasite Core Facilities of the Johns Hopkins Malaria Research Institute. This work was supported by a grant R01AI031478 from the National Institutes of Health, by the NIH Distinguished Scholars Program, and the Intramural Research Program of the Division of Intramural Research AI001250-01, National Institutes of Allergy and Infectious Diseases, National Institutes of Health and by the Bloomberg Philanthropies. Supply of human blood was supported by the National Institutes of Health grant RR00052. We thank Dr. Yuemei Dong from the Johns Hopkins Malaria Research Institute for providing the helper plasmid and Dr. Christopher Potter from Johns Hopkins School of Medicine for providing pXL-BACIIECFP15XQUAS-TATA-PAI-SV40 and pXL-BACII-DsRed-AAPP-QF2-hsp70 plasmids. 

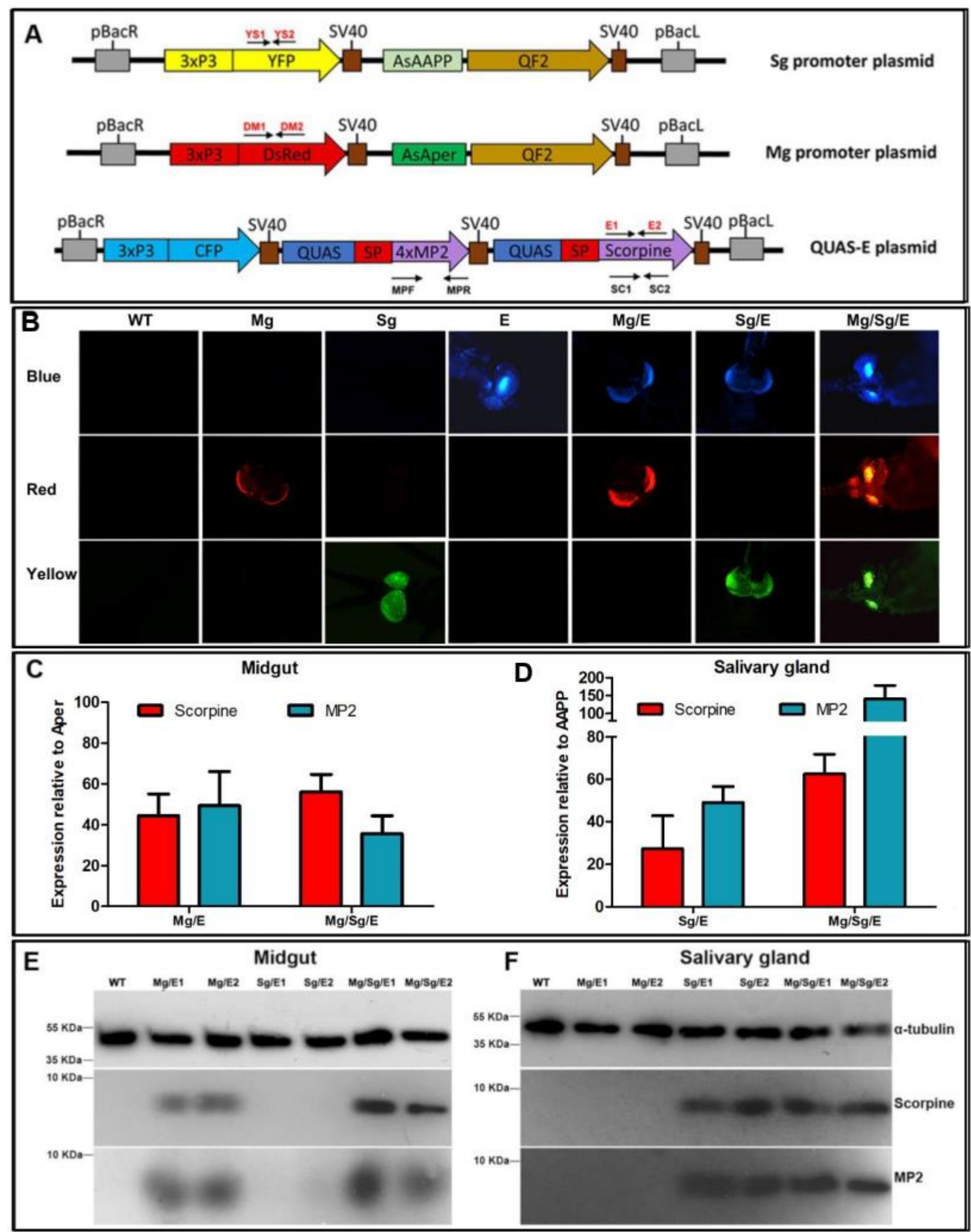
669 [A]: Diagram of the salivary gland $(\mathrm{Sg})$ and midgut $(\mathrm{Mg})$ driver constructs expressing the QF2 670 transcription factor and of the effector (E) constructs expressing the MP2 and scorpine effector

671 proteins under control of the QUAS promoter. Each construct also includes sequences encoding a 672 yellow (YFP), red (DsRed) or blue (CFP) fluorescent protein under the control of the 3xP3 eye 673 promoter. pBac: piggyBac inverted terminal repeats; SV40: transcription terminator sequence; 674 SP: An. stephensi carboxypeptidase signal peptide. Primers used for validation of insertion into 675 mosquito lines (Figure S1 and Table S7) are indicated in red font. Primers used for qRT-PCR are 676 indicated in black font (Table S7). [B] Detection of fluorescent eye markers in wild type (WT) 677 and transgenic mosquitoes carrying different combinations of midgut driver $(\mathrm{Mg})$, salivary gland 678 driver (Sg) and effector (E) sequences. Tissue-specific expression of MP2 and scorpine mRNA 679 in transgenic mosquitoes quantified by qRT-PCR in the midgut relative to the endogenous Aper 680 mRNA [C], and in the salivary glands relative to the endogenous AAPP mRNA [D]. Mosquito $681 \mathrm{rpS7}$ was used as a reference. Data pooled from three independent biological replicates. 682 Statistical analysis was determined by the Student's t test. [E] and [F] Immunoblotting showing 683 MP2 $(6.17 \mathrm{kDa})$ and scorpine peptide $(8.46 \mathrm{kDa})$ protein expression in midgut and salivary gland 684 lysates from wild type and transgenic lines. $\alpha$-tubulin was used as a loading control. E1 and E2 685 refer to independent mosquito transgenic lines. Antibodies used are shown to the right of [F]. 
A

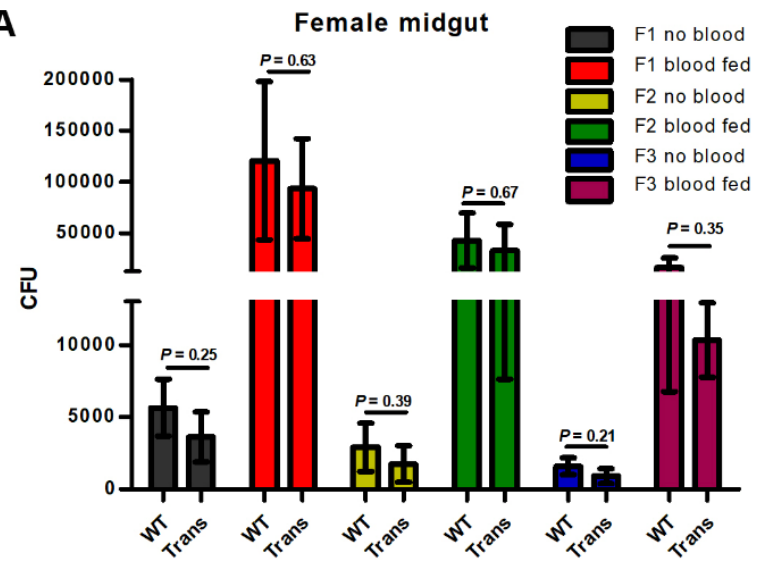

C

Male midgut

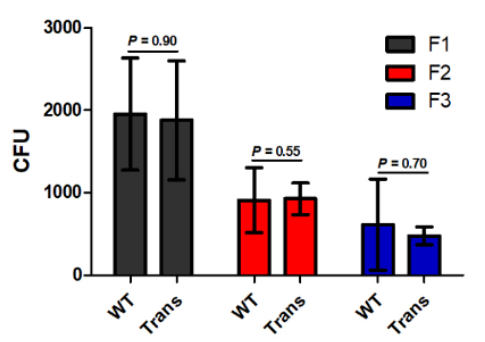

D

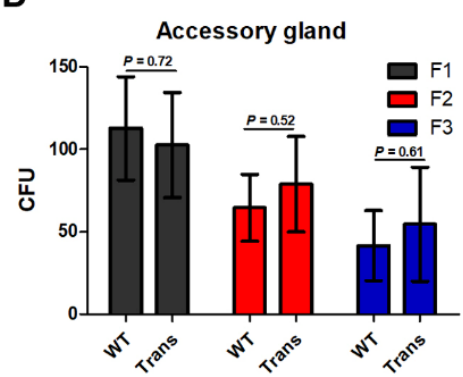

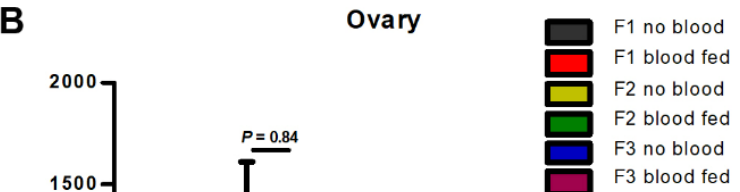

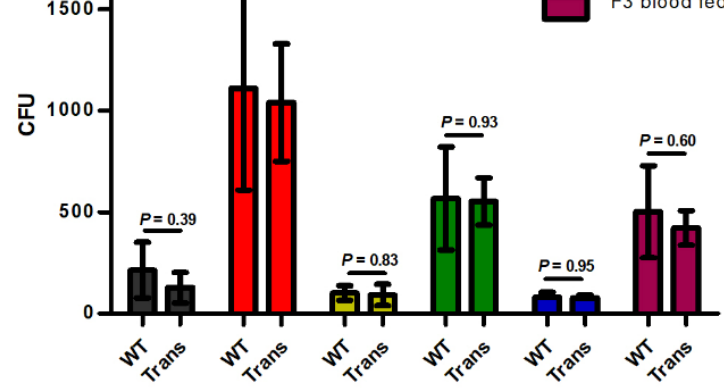

E Serratia transmission - male to virgin female

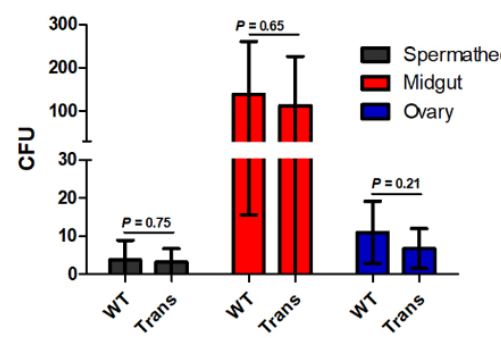

Figure 2 Serratia $A S 1$-poly-effector bacteria persist through multiple mosquito generations. A total of $100 \mathrm{WT}$ or transgenic (Trans) virgin females that had been fed with AS1-poly-effector bacteria were placed in a cage with $100 \mathrm{WT}$ or transgenic virgin males (not fed with bacteria) and allowed to mate. Mosquitoes were then fed blood and allowed to lay eggs. These eggs were allowed to hatch and reared to adults following standard protocol (F1). The F1 mosquitoes were propagated through two additional generations (F2 \& F3) without providing additional genetically modified bacteria. At each generation, 10 mosquitoes were dissected, and bacterial load was determined by plating serial dilutions of tissue homogenates on apramycin and ampicillin agar plates and counting colonies. [A] Colony-forming units (CFUs) per female midgut fed or not on blood. [B] CFUs per female ovary fed or not on blood. [C] CFUs per male midgut. [D] CFUs per male accessory gland. Data pooled from 3 independent experiments. [E] Serratia horizontal (sexual) transmission. Newly emerged virgin male adult mosquitoes were fed on 5\% sugar solution containing $10^{7} / \mathrm{ml} A S 1$-poly-effector bacteria $/ \mathrm{ml}$ and then allowed to mate with virgin females. Three days later, 10 females were assayed for the presence of Serratia AS1 by plating spermatheca, midgut and ovary homogenates on apramycin and ampicillin agar plates and counting colonies. Trans: $\mathrm{Mg} / \mathrm{Sg} / \mathrm{E}$ transgenic mosquitoes. Error bars indicate standard deviation of the mean. Data pooled from 3 independent biological experiments. Statistical analysis was determined by the Student's t test. $P>0.05$ : not significant. 
A

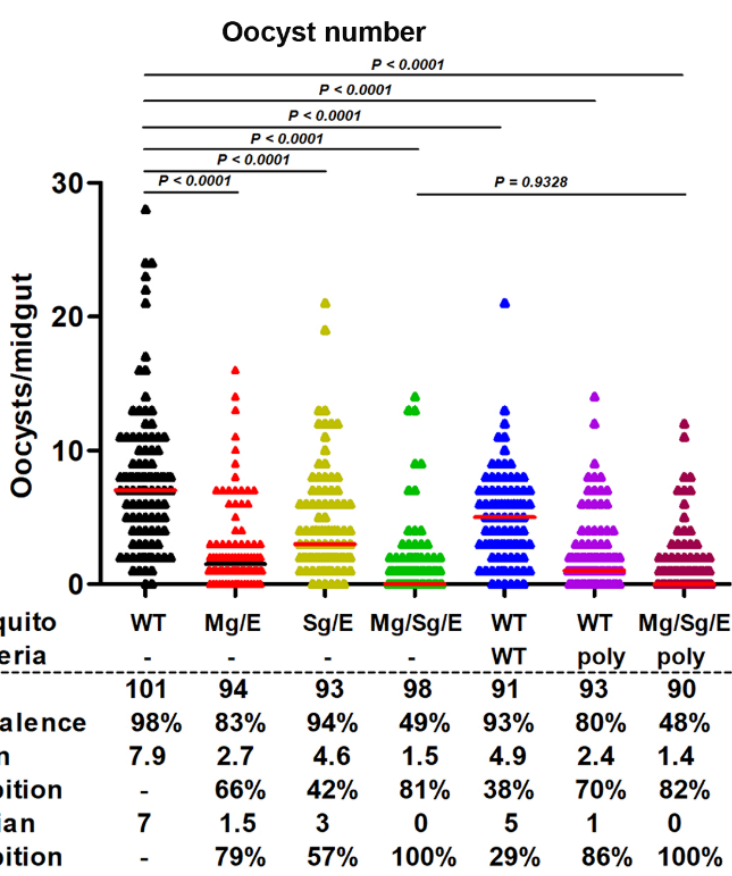

B

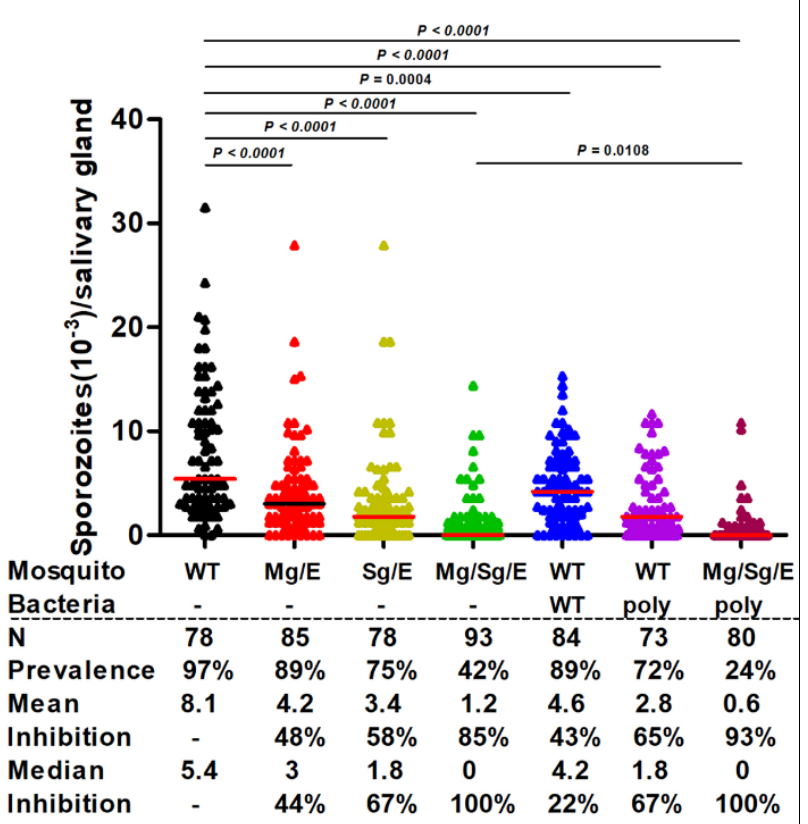

Figure 3 Transgenesis and paratransgenesis strongly impair Plasmodium development.

710 Two-day-old An. stephensi mosquitoes were fed (or not) overnight with wild type or recombinant

711 Serratia AS1 (poly) bacteria, as indicated. After 48 h, all mosquito groups were fed on the same

712 P. falciparum gametocyte culture and midgut oocyst number was determined on day 7 [A] and

713 salivary gland sporozoite number was determined on day 14 [B] post-feeding. Horizontal red

714 lines represent median oocyst or sporozoite number. Data pooled from three independent

715 biological experiments. Statistical analysis by Mann-Whitney U test. $P>0.05$ : not significant;

716 'poly': Serratia AS1-poly bacteria; N: number of mosquitoes assayed; Prevalence: proportion of

717 mosquitoes carrying one or more parasite. 

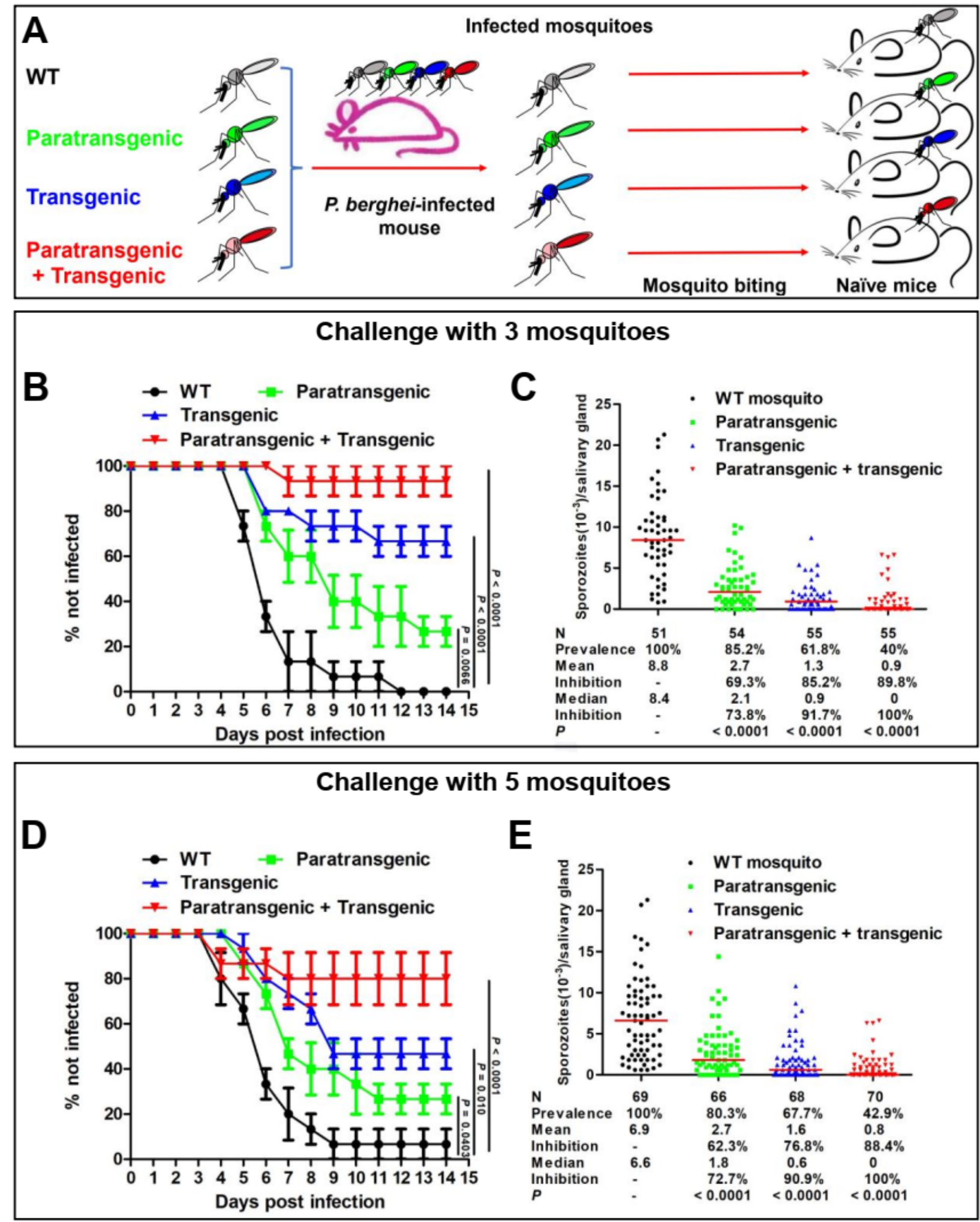

Figure 4 Transgenesis and paratransgenesis inhibit $P$. berghei transmission by mosquitoes

from infected to naïve mice. [A] Experimental design. Wild type (WT), paratransgenic, 
723 transgenic and [paratransgenic + transgenic] mosquitoes were fed on the same P. berghei-

724 infected mouse, assuring that all mosquitoes ingested the same number of parasites. After 21 23

725 days, when sporozoites had reached the salivary glands $[\mathbf{C}, \mathbf{E}]$, three $[\mathbf{B}]$ or five $[\mathbf{D}]$ mosquitoes

726 were randomly selected and allowed to bite naïve mice. The parasitemia of these mice was

727 followed for 14 days. Data pooled from three independent experiments, each using five mice per

728 challenged group for a total of 15 mice. Transgenic mosquitoes express effectors in both midgut

729 and salivary glands. Data pooled from three independent biological experiments. Statistical

730 analysis (B and D) was determined by the Log-rank (Mantel-Cox) test; Statistical analysis (C and

731 E) was determined by the Mann-Whitney U test. 


\section{SUPPLEMENTARY FIGURES}

733
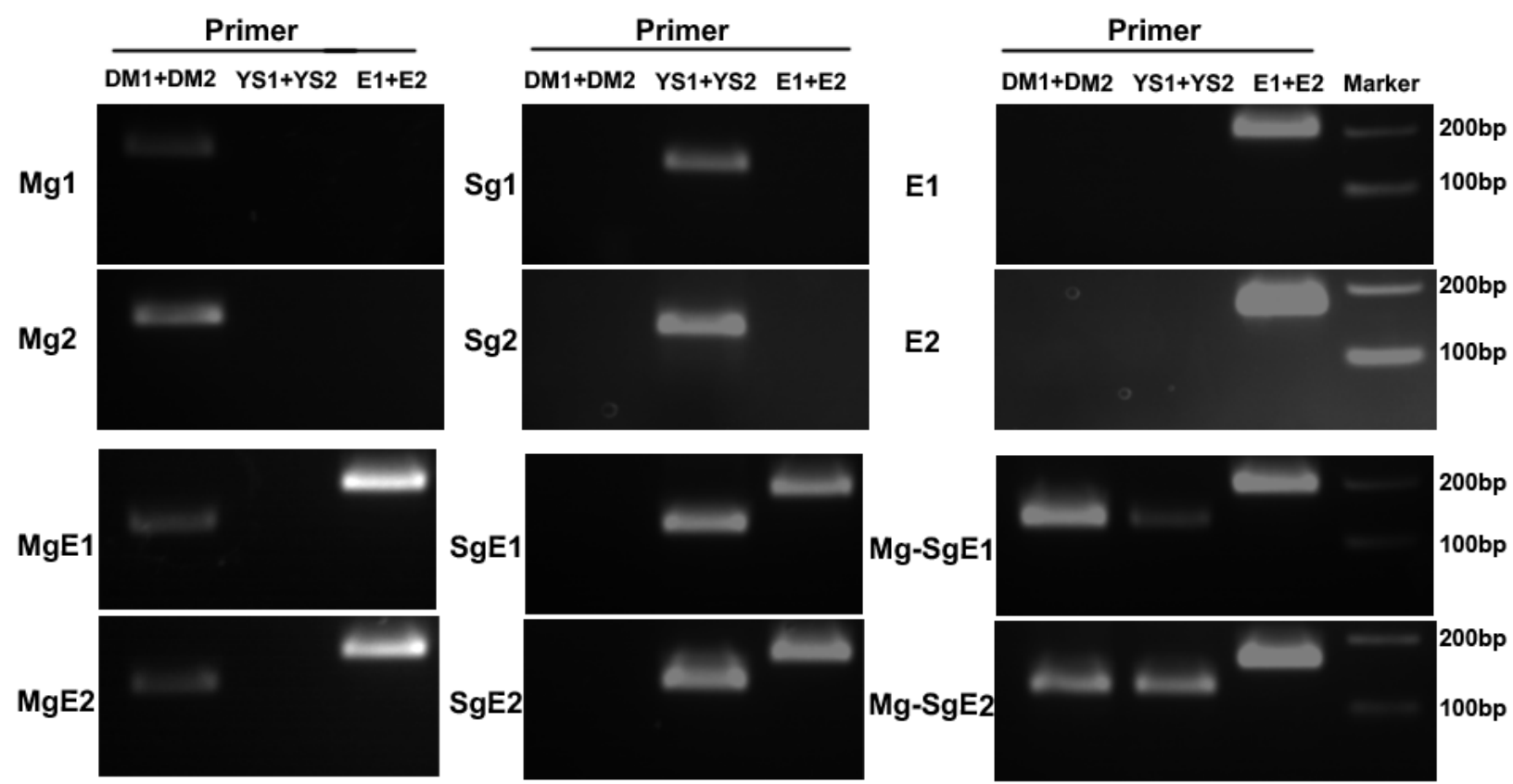

Figure S1 PCR validation of plasmid insertion site in mosquito lines. Primer pairs used for

PCR reactions are indicated on top of each lane (sequences provided in Table S7; position of primers indicated in Figure 1A with red font). The DM1+DM2 primer pair was used to verify the MG QF2 driver plasmid insertion; the YS1+YS2 primer pair was used to verify the SG QF2 driver plasmid insertion; and the E1+E2 primer pair was used to verify the QUAS-MP2-QUASscorpine effector plasmid insertion. The transgenic lines are identified to the left of each panel. 

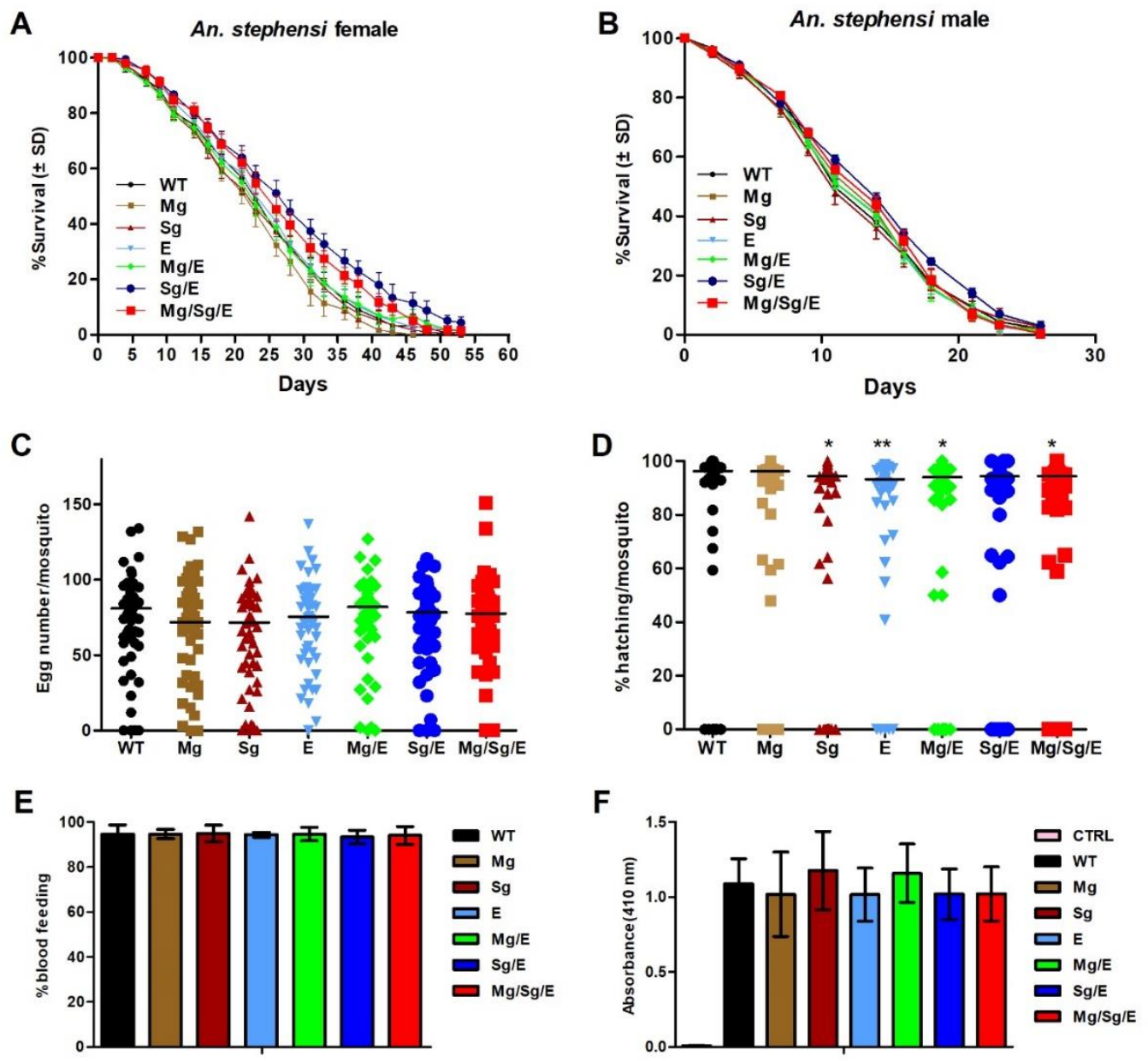

Figure S2 Fitness analysis of $\boldsymbol{A n}$. stephensi transgenic lines. [A and B]: Survival curves for WT and transgenic (see Figure 1A) females that received one blood meal on day two [A] and males [B], all maintained on sugar meal. No significant differences in survival rate were detected as calculated by Kaplan-Meier survival curves, and multiple comparisons by Log-rank test with Bonferroni correction for parental expressing lines. For [A] WT \& Mg: $P=0.99$; WT \& Sg: $P=$ 0.65 ; WT \& E: $P=0.46$; WT \& Mg/E: $P=0.64$; WT \& Sg/E: $P=0.32$; WT \& Mg/Sg/E: $P=$ 0.37 and for [B] WT \& Mg: $P=0.66$; WT $\& \mathrm{Sg}: P=0.53$; WT $\& \mathrm{E}: P=0.99$; WT $\& \mathrm{Mg} / \mathrm{E}: P=$ 0.97; WT \& Sg/E: $P=0.075$; WT \& $\mathrm{Mg} / \mathrm{Sg} / \mathrm{E}: P=0.34]$. Combined from three biological replicates (N: 300 mosquitoes). [C]: Comparison of fecundity (number of laid eggs) between WT and transgenic mosquitoes. No significant differences were found using the Log-rank (Mantel-Cox) test. WT \& Mg: $P=0.74$; WT \& Sg: $P=0.24$; WT \& E: $P=0.77$; WT $\&$ Mg/E: $P$ $=0.70 ; \mathrm{WT} \& \mathrm{Sg} / \mathrm{E}: P=0.80 ; \mathrm{WT} \& \mathrm{Mg} / \mathrm{Sg} / \mathrm{E}: P=0.97$ [D]: Comparison of fertility (proportion of laid eggs that hatched) between WT and transgenic lines. Statistical analysis used the MannWhitney U test. WT \& Mg: $P=0.93$; ${ }^{*} \mathrm{WT} \& \mathrm{Sg}: P=0.021 ; * * \mathrm{WT} \& \mathrm{E}: P=0.0089$; *WT \& $\mathrm{Mg} / \mathrm{E}: P=0.032 ; \mathrm{WT} \& \mathrm{Sg} / \mathrm{E}: P=0.050 ;{ }^{*} \mathrm{WT} \& \mathrm{Mg} / \mathrm{Sg} / \mathrm{E}: P=0.032$ ]. [C and D]: data combined from three biological replicates (N: 60 mosquitoes); horizontal lines are median values. [E] The percentage of mosquitoes that take a blood meal is not affected. Two-day-old 
761 female mosquitoes were allowed to feed on mice, and the percentage of mosquitoes that fed was 762 determined after 30 min feeding. WT \& Mg: $P=1.00$; WT \& Sg: $P=0.92$; WT \& E: $P=0.90$;

763 WT \& Mg/E: $P=1.00 ; \mathrm{WT} \& \mathrm{Sg} / \mathrm{E}: P=0.68 ; \mathrm{WT} \& \mathrm{Mg} / \mathrm{Sg} / \mathrm{E}: P=0.85$. [F] The amount blood 764 uptake is not affected. Quantification of protein-bound heme at $410 \mathrm{~nm}$ from midguts of WT and 765 transgenic mosquitoes before (CTRL) and after a blood meal. WT \& Mg: $P=0.73$; WT \& Sg: $P$ $766=0.65 ; \mathrm{WT} \& \mathrm{E}: P=0.63 ; \mathrm{WT} \& \mathrm{Mg} / \mathrm{E}: P=0.66 ; \mathrm{WT} \& \mathrm{Sg} / \mathrm{E}: P=0.64 ; \mathrm{WT} \& \mathrm{Mg} / \mathrm{Sg} / \mathrm{E}: P=$ 767 0.76. [E and F]: error bars represent SD of the mean; data pooled from three independent 768 experiments; no significant differences were found using the Student's t test. 
A

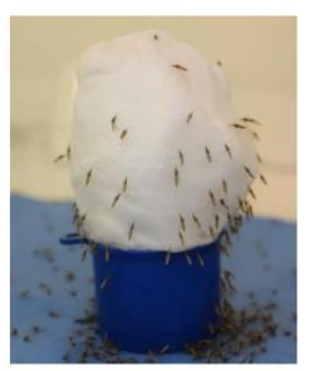

1) Feed Serratia to mosquitoes

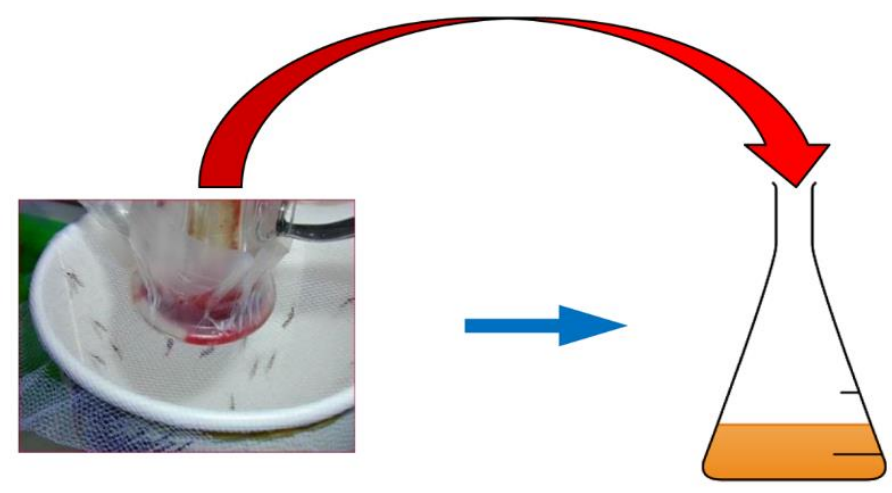

3) Culture blood from feeder in LB overnight

B

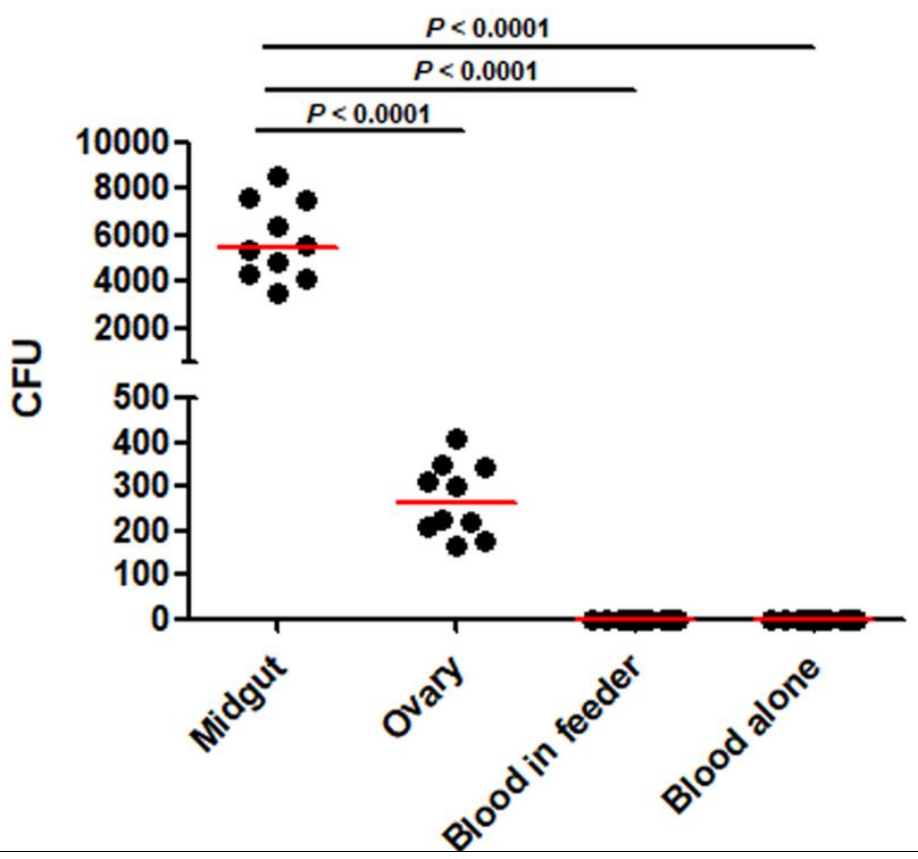
2) Feed blood to
mosquitoes

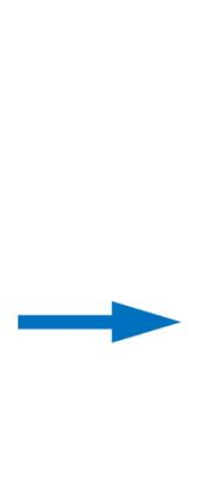

\section{B}


bioRxiv preprint doi: https://doi.org/10.1101/2022.03.02.482642; this version posted March 2, 2022. The copyright holder for this preprint (which was not certified by peer review) is the author/funder, who has granted bioRxiv a license to display the preprint in perpetuity. It is made available under aCC-BY 4.0 International license.

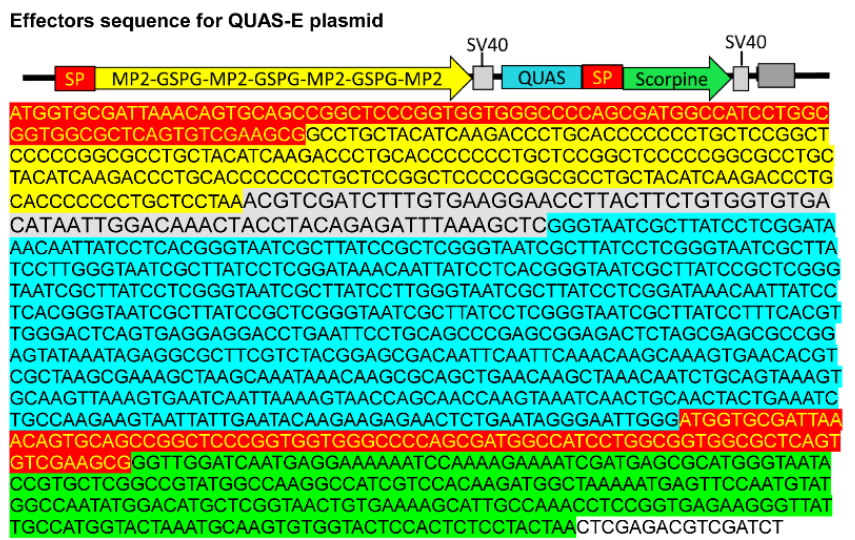

YFP

ATGGTGAGCAAGGGCGAGGAGCTGTTCACCGGGGTGGTGCCCATCCTGGTCGAGCTGGACGG CGACGTAAACGGCCACAAGTTCAGCGTGTCCGGCGAGGGCGAGGGCGATGCCACCTACGGCA AGCTTTCGCGTCGGTAGTAGGACCCTGAAGTTCATCTGCACCACCGGCAAGCTGCCCGTGCCC TGGCCCACCCTCGTGACCACCTTCGGCTACGGCGTGCAGTGCTTCGCCCGCTACCCCCGACCAC ATGCGCCAGCACGACTTCTTCAAGTCCGCCATGCCCGAAGGCTACGTCCAGGAGCGCACCATC TTCTTCAAGGACGACGGCAACTACAAGACCCGCGCCGAGGTGAAGTTCGAGGGCGACACCCT GGTGAACCGCATCGAGCTGAAGGGCATCGACTTCAAGGAGGACGGCAACATCCTGGGGCACAA GCTGGAGTACAACTACAACAGCCACAACGTCTATATCATGGCCGACAAGCAGAAGAACGGCATC AAGGTGAACTTCAAGATCCGCCACAACATCGAGGACGGCAGCGTGCAGCTCGCCGACCACTAC CAGCAGAACACCCCCATCGGCGACGGCCCCGTGCTGCTGCCCGACAACCACTACCTGAGCTAC CAGTCCGCCCTGAGCAAAGACCCCAACGAGAAGCGCGATCACATGGTCCTGCTGGAGTTCGTG ACCGCCGCCGGGATCACTCTCGGCATGGACGAGCTGTACAAGTAG

AsAAPP (anopheline antiplatelet protein gene) promoter

GGACTTCGCGTCGGTAGTAGTATTCTCCGGCAACGCTTTCCCAACCGTGATCGCGAAGTCCCTC GCCACAACAGCTTGCCTCAGCCGATCCGTATTGAGCCTAGAAGTAGGCTGATAGCGCTGCGTAT TGGCTACGCAGAGCTTTTGGCGTAACTTTACCATAACCAGGACATGAGTTGACTTTTGCTCCTCT GTAGGTCCGTAGGCCGGTTATATCCGAGAAGTGCCTCCCATCGATGAGAACGAGGTCAATCTGG GAATATGTCTGCTGTGGTGATCTCCAGGTGTAGCTGAACCGAGGTGCATCATCAAGAGCTCATT GTCGTTCGTCAGCGGGTGGGTGCTGAAACTTCCTATTGTGGGTTTGTATGCCTCCTCCCGTCCG

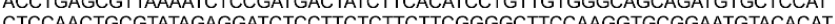
CTCCAACTGCGTATAGAGGATCTCCTTCT TOT TAATAATGCTGAGGTTGAAGAACCTGCCACGGATCCTCAGCCTGCACCGTAAATGATCTGCAGTT

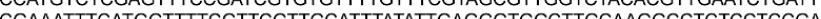
CGAAATTCATGTT GTTCGTCATTATAT AATCTATCTCGTCGGAGGCCTACGCTITATCTATA GCCAGCCGCCCCTAACATCCAGAACAGAGGCTAGCTATACGCCCCCCCCCCCCCCCTCAATTA GACATACCATTCTGTATGCCGGGGACGTATTGTGTCTAAGTGGGGTGTGGAGAGCCTATGTTAAC GACATACCATTCTGTATGCCGGGGACGTATGTGTCTAAGTGGGGTGTGGAGATTCTATGTTAAC ATCATCTGCITCTACCATCCCTACTATATACAATATGGGAGATGGTTATATATTGGAAA GTATGTG ATTTT GTC GT CCAAGTA CACCACAGTGAAACAAGTGTAATAAGAGTAAATTTGATTATTTAATATTTATTTACCTTCGCATAA CACCGCCAGTGAAACAAGTGTAATAAGAGTAAAAT TIGATTATIAATATTTAT TACCTTCGCATAA TTACT TTACTTAATGATCATCGTATTGCAACATGCTTGTTAACCATTATT TATCAAACATTACTTTCCCTC TACTITGCACAGTAAACAGTGCATTGCTTAACACTAACAAAAGCTGTACAAATACTAGGG AGATCATCAGTAGAGTTTGCCTTTTTTCCATAGCTCACAGGTGAATAAACG

AsAper1 (adult peritrophic matrix protein 1 gene) promoter

TACCGGCAATACTGGTTGTTGAGGGGAAAAAATAGACAATAAAATTTTTCTTTCAGCTTTGAATGA TGGATAATGCTTTATTTCGGTTCAGGAGCTTGTATTGCTTCGCTGGATAACTGGAACTTGGAATCA ACGTTGTACAGGTTGTAAATGTTCCTCAAGCCCTGGAAAAAGTGTCTACATCTTCAAAACGATGA GCGATTTTGATATAAAGACAACAGTTTTGGGGGTGACCGGAGCGATAACTTTCTTTAACTTTAACT GCGATTCTTTCGAGATTATAGAACCAAAGAAGAAGATATCAATTTTGATATAATCGTACATGCTTTTAA ACATAAAGACACTTTCTTATGATCTAAGAAACACTGTACACACACTTATTACATGCCGTCCAATAT ACATAAAGACACTTTCTTTATGATCTAAGAAACACTGTACACACACTTATTACATGCCGTCCAATAT TGTTCAGTGCTTAAGTATTGAGGTTAATTGTTAATGTTAGAATAAAGCCTTCTACACTACACGGCAA GGAGTGTAAAGCAGTGATGATGATGATGGTGATTATTCGGATTATCAAGTTGATAAAGAATTAGCT ACATCAATTGGTTACAGCAGAATAATCACTTTATACCAATATGATGTCACCATCAAGCGTATCGACA CAAACTGTCTGTCTCACTCTCTTGTGTTCTACCAATTTGGGGACCAATGCCATAGGTGTCCTGCC CCGGGGTGTGTCTGCCCCCTGACCGGGACACATGTCCGTTGCGAGTGTTTTATAAACGCGCGC ACACCCAATAACCATAATCGTAACGATGAACGGCTTGTGTGCCTATCGGAAAAGCGTCTGCTTTT TTTGGGAGGGCTGCTTCAATTGATGCAGTTAAAAAGGGAACGAGCGTGATGATTATGATTACAAC TAATATGCATATGAGTGGAGCCGTGTGGAGGATAATCCACGCCACACTCTGGTGGATGCGTCCG ATGGAGACACTTTAAATAAATGGCCAAATTTGCAGTTCATAAAATCGAATGAAGGATAACCAGAAT GAACGTTAGG GATCGTATTG GATA CAAAGTTAGTCATCAGCAGACAGCTTTAGTAGATCGCCAAATAGACACGAGCACCGTGCTGAGAT TCACTTTTGATAGCCGATAGTCGATAAGTTGAAGTGTCCCACCATCCAAGCAGCGTGCCAGTTA AATCAACTGTACACCGTTICGAGAGGCAGCGATGGACAGTGCTTAAAATGTTGCTAACAGTCAGT CCACTTCAGATTATCCATGCTATCGGAACCGTAGCGCCTCCAATAAAAAGGACACACCGGACGGT
GCAACAGGTACAGTACGTAACGTGCGTTGACGGTAATACATACAACTCGCGGATCTAACATTCT CATC 
Table S1. Transgene integration sites

788

\begin{tabular}{|c|c|c|}
\hline & INTEGRATION SITE & $\begin{array}{c}\text { INTEGRATION } \\
\text { IN GENE }\end{array}$ \\
\hline \hline LINE & AsteS1:KB664810:1:1229869:1 & NO \\
\hline Mg2 & AsteS1:KB664721:1:1159608:1 & NO \\
\hline Sg1 & AsteS1:KB664422.1 & NO \\
\hline AsteS1:KB664506.1 & $\begin{array}{c}\text { Gamma- } \\
\text { AsteS1: KB664514.1 }\end{array}$ & $\begin{array}{c}\text { (ASTE010947) } \\
\text { glutamyltranspeptidase } \\
\end{array}$ \\
\hline E1 & AsteS1: KB664921.1 & NO \\
\hline E2 & AsteS1:KB664810:1:1229869:1 & NO \\
\hline & AsteS1:KB664810:1:1229869:1 & NO \\
& AsteS1:KB664538:1:382792:1 & NO \\
\hline
\end{tabular}

790 Integration site: AsteS1: contig number: precision site 
Table S2. Verification of transgene homozygosity

\begin{tabular}{|c|c|c|c|c|}
\hline MOSQUITO LINE & $\begin{array}{c}\text { LARVA } \\
\text { NUMBERS } \\
\end{array}$ & RED & YELLOW & BLUE \\
\hline Mg1 & 412 & 412 & & \\
\hline Mg2 & 276 & 276 & & \\
\hline Sg1 & 178 & & 178 & \\
\hline Sg2 & 329 & & 329 & \\
\hline E1 & 206 & & 206 & \\
\hline E2 & 378 & & 378 & \\
\hline $\mathbf{M g} / \mathbf{E} 1$ & 262 & 262 & & 262 \\
\hline $\mathbf{M g} / \mathbf{E 2}$ & 198 & 198 & & 198 \\
\hline $\mathrm{Sg} / \mathrm{E} 1$ & 307 & & 307 & 307 \\
\hline $\mathrm{Sg} / \mathrm{E} 2$ & 345 & & 345 & 345 \\
\hline $\mathrm{Mg} / \mathrm{Sg} / \mathrm{E} 1$ & 228 & 228 & 228 & 228 \\
\hline $\mathrm{Mg} / \mathrm{Sg} / \mathrm{E} 2$ & 361 & 361 & 361 & 361 \\
\hline
\end{tabular}

796

797
A total of 20 transgenic female mosquitoes from each line were mated with wild type males and the progeny larvae assayed for expression of the dominant eye fluorescence marker. No notfluorescent larvae were found, indicating that the females were homozygous for the transgenes. 
800

801

802

803

804
Table S3. Expression of MP2 and scorpine mRNAs relative to the endogenous AsAper mRNA, quantified by qRT-PCR in the midgut of transgenic mosquitoes.

\begin{tabular}{|c|c|c|c|}
\hline \multirow{2}{*}{ Mosquito lines } & \multicolumn{3}{|c|}{ Relative expression in midgut } \\
\cline { 2 - 4 } & AsAper & Scorpine & MP2 \\
\hline $\mathrm{E}$ & $1.0 \pm 0.1$ & $\mathrm{~N}$ & $\mathrm{~N}$ \\
\hline $\mathrm{Mg} / \mathrm{E}$ & $1.0 \pm 0.3$ & $44.3 \pm 10.7$ & $49.3 \pm 16.7$ \\
\hline $\mathrm{Mg} / \mathrm{Sg} / \mathrm{E}$ & $1.0 \pm 0.2$ & $56.1 \pm 8.7$ & $35.6 \pm 8.9$ \\
\hline
\end{tabular}

The rpS7 gene was used as reference, and WT mosquitoes were used as negative controls. Identification of mosquito lines provided in Figure 1A. N: transcript not detected. Data pooled from three independent biological experiments. Mean \pm SD. 
807

\section{8}

809

810

811

812
Table S4. Relative expression of MP2 and scorpine mRNAs relative to the endogenous AsAAPP mRNA quantified by qRT-PCR in the salivary glands of transgenic mosquitoes.

\begin{tabular}{|c|c|c|c|}
\hline \multirow{2}{*}{ Mosquito lines } & \multicolumn{3}{|c|}{ Relative expression in salivary gland } \\
\cline { 2 - 4 } & AsAAPP & Scorpine & MP2 \\
\hline \hline $\mathrm{E}$ & $1.0 \pm 0.2$ & $\mathrm{~N}$ & $\mathrm{~N}$ \\
\hline $\mathrm{Sg} / \mathrm{E}$ & $1.0 \pm 0.1$ & $27.3 \pm 15.6$ & $49.1 \pm 7.6$ \\
\hline $\mathrm{Mg} / \mathrm{Sg} / \mathrm{E}$ & $1.0 \pm 0.3$ & $62.5 \pm 9.3$ & $140.2 \pm 38.3$ \\
\hline
\end{tabular}

The rpS7 gene was used as reference, and WT mosquitoes were used as negative controls. Identification of mosquito lines provided in Figure 1A. N: transcript not detected. Data pooled from 3 independent experiments. Mean \pm SD. 
Table S5. Serratia is horizontally (sexually) transmitted.

814

\begin{tabular}{|c|c|c|c|c|}
\hline \multirow{2}{*}{$\begin{array}{c}\text { Males carrying } \\
\text { AS1-poly }\end{array}$} & $\begin{array}{c}\text { Females } \\
\text { (mated/virgin) }\end{array}$ & Spermatheca & Midgut & Ovary \\
\cline { 3 - 5 } & WT mated & 0 & 0 & 0 \\
\hline \hline WT & Transgenic mated & 0 & 0 & 0 \\
\hline Transgenic & WT virgin & $3.9 \pm 4.7$ & $115 \pm 118$ & $11 \pm 7.7$ \\
\hline WT & Transgenic virgin & $2.9 \pm 4.7$ & $104 \pm 111$ & $8.7 \pm 7.8$ \\
\hline Transgenic & & & & \\
\hline
\end{tabular}

815

816

817

818

819

820

821
Newly emerged virgin male adult mosquitoes were fed overnight on 5\% sugar solution containing $10^{7} \mathrm{AS} 1$-poly bacteria/ml and placed with females. Three days later, 10 females were assayed for the presence of Serratia $A S 1$ by plating midgut, ovary and spermatheca homogenates on apramycin/ampicillin agar plates and colonies were counted. Transgenic mosquito: $\mathrm{Mg} / \mathrm{Sg} / \mathrm{E}$. Female mosquitoes mate only once in their lifetimes; mated females were used as controls. Data pooled from 3 independent experiments. Mean \pm SD. 
bioRxiv preprint doi: https://doi.org/10.1101/2022.03.02 482642; this version posted March 2, 2022. The copyright holder for this preprint (which was not certified by peer review) is the author/funder, who has granted bioRxiv a license to display the preprint in perpetuity. It is made available under aCC-BY 4.0 International license.

Table S6. Vectors used in this research.

823

\begin{tabular}{cc}
\hline Vectors & Reference/notes \\
\hline \hline phsp-pBac & 33 \\
\hline pXL-BACII-DsRed-AAPP-QF2-hsp70 & 15 \\
\hline pXL-BACIIECFP-15XQUAS-TATA-PAI- \\
SV40 & 15 \\
\hline pXL-BACII- DsRed-Aper-QF2-Hsp70 & Mg QF2 driver plasmid \\
\hline $\begin{array}{c}\text { pXL-BAC-YFP-AAPP promoter-QF2-Hsp70 } \\
\text { SXL-BACIIECFP-15XQUAS-TATA-MP2- }\end{array}$ & QUAS-MP2-Scorpine effector plasmid \\
\hline pBAM2-YFP & DNA template for YFP \\
\hline
\end{tabular}

824 
Table S7. Oligonucleotide primers used in this study.

\begin{tabular}{|c|c|c|}
\hline Primer & Sequence (5'- 3') & Notes \\
\hline $\mathrm{MgPF}$ & ATCAATGTATCTCGAGTACCGGCAATACTGGTTGTTGAGG & \multirow{2}{*}{$\begin{array}{l}\text { MgPF and MgPR to } \\
\text { amplify midgut } \\
\text { promoter which was } \\
\text { insert to construct } \\
\text { MG QF2 driver } \\
\text { plasmid, restriction } \\
\text { site XhoI }\end{array}$} \\
\hline $\mathrm{MgPR}$ & GTTGGCCGGCCTCGAGGATGAGAATGTTAGATGCCGCGAGTTG & \\
\hline YFPF & GGGCCCGGGATCCACCGGTCGCCACCATGGTGAGCAAGGGCGAGGA & \multirow{4}{*}{$\begin{array}{l}\text { YFPF and YFPR to } \\
\text { amplify YFP which } \\
\text { was inserted to ApaI } \\
\text { and NotI sites, then, } \\
\text { SgPF and SgPR to } \\
\text { amplify salivary } \\
\text { gland promoter } \\
\text { which was inserted } \\
\text { to construct SG QF2 } \\
\text { driver plasmid at } \\
\text { site XhoI }\end{array}$} \\
\hline YFPR & GCGGCCGCTACTTGTACAGCTCGTCCA & \\
\hline $\mathrm{SgPF}$ & ATCAATGTATCTCGAGGGACTTCGCGTCGGTAGTAG & \\
\hline $\mathrm{SgPR}$ & GTTGGCCGGCCTCGAGCGTTTATTCACCTGTGAGCTATGG & \\
\hline $\begin{array}{c}\text { MP2- } \\
\text { ScopineF }\end{array}$ & GCGGCCGCGGCTCGAGATGGTGCGATTAAACAGTGCA & \multirow{2}{*}{$\begin{array}{l}\text { MP2-ScopineF and } \\
\text { MP2-ScopineR to } \\
\text { amplify effectors } \\
\text { genes which were } \\
\text { inserted to construct } \\
\text { QUAS-E plasmid, } \\
\text { restriction site XhoI }\end{array}$} \\
\hline $\begin{array}{c}\text { MP2- } \\
\text { ScopineR }\end{array}$ & AGATCGACGTCTCGAGTTAGTAGGAGAGTGGAGTAC & \\
\hline AAPPF & GTACGAAGAGTGCAGCAAGG & \multirow{2}{*}{$\begin{array}{l}\text { For RT-PCR: } \\
\text { AsAAPP gene }\end{array}$} \\
\hline AAPPR & TCGATGAGTCCCTCGTCAAG & \\
\hline PorF & AATGACTCCCAGAAGCAGTG & \multirow{2}{*}{$\begin{array}{l}\text { For RT-PCR: } \\
\text { AsAper1 gene }\end{array}$} \\
\hline PorR & ACTTCACTCTTCACACTGCG & \\
\hline $\mathrm{SC} 1$ & GCGGGTTGGATCAATGAG & \multirow{2}{*}{$\begin{array}{l}\text { For RT-PCR: } \\
\text { scorpine gene }\end{array}$} \\
\hline $\mathrm{SC} 2$ & AGTTAGTAGGAGAGTGGA & \\
\hline MPF & GTCGAAGCGGCCTGCTAC & \multirow{2}{*}{$\begin{array}{l}\text { For RT-PCR: MP2 } \\
\text { gene }\end{array}$} \\
\hline MPR & AGATCGACGTTTAGGAGC & \\
\hline S7F & CTAACGACACGAAGACCACAAGA & \multirow{2}{*}{$\begin{array}{l}\text { For RT-PCR: S7 } \\
\text { gene }\end{array}$} \\
\hline S7R & CAACCTGCAACGAAGCAAAA & \\
\hline
\end{tabular}


bioRxiv preprint doi: https://doi.org/10.1101/2022.03.02 482642; this version posted March 2, 2022. The copyright holder for this preprint (which was not certified by peer review) is the author/funder, who has granted bioRxiv a license to display the preprint in perpetuity. It is made available under aCC-BY 4.0 International license.

\begin{tabular}{|c|c|c|}
\hline YS1 & AGGACCCTGAAGTTCATCTG & \multirow{4}{*}{$\begin{array}{l}\text { For verification of } \\
\text { SG QF2 driver } \\
\text { plasmid insertion } \\
\text { For verification of } \\
\text { MG QF2 driver } \\
\text { plasmid insertion }\end{array}$} \\
\hline YS2 & CTTCGGGCATGGCGGACTTG & \\
\hline DM1 & GTGAACTTCCCCTCCGACG & \\
\hline DM2 & TCAGCTTCAGGGCCTTGTG & \\
\hline E1 & AAAATCCAAAAGAAAATCGATGAGC & \multirow{2}{*}{$\begin{array}{l}\text { For verification of } \\
\text { QUAS-MP2-QUAS } \\
\text { scorpine effector } \\
\text { plasmid insertion }\end{array}$} \\
\hline E2 & GAGTGGAGTACCACACTTGCAT & \\
\hline SPLNK\#1 & CGAAGAGTAACCGTTGCTAGGAGAGACG & \multirow{4}{*}{$\begin{array}{l}\text { Primers for } \\
\text { Splinkerette PCR }\end{array}$} \\
\hline SPLNK\#2 & GTGGCTGAATGAGACTGGTGTCGAC & \\
\hline pBacRE\#1 & CGATATACAGACCGATAAAACACATGCGTC & \\
\hline pBacLE \#2 & GCGACTGAGATGTCCTAAATGCAC & \\
\hline
\end{tabular}


bioRxiv preprint doi: https://doi.org/10.1101/2022.03.02 482642; this version posted March 2, 2022. The copyright holder for this preprint (which was not certified by peer review) is the author/funder, who has granted bioRxiv a license to display the preprint in perpetuity. It is made available under aCC-BY 4.0 International license.

Table S8. Plasmid injection and screening for transformants

830

\begin{tabular}{|lc|c|c|c|c|}
\hline \multicolumn{1}{|c|}{ Donor plasmid } & Helper & $\begin{array}{c}\text { \# embryos } \\
\text { injected }\end{array}$ & $\begin{array}{c}\text { \# Go (number } \\
\text { of survivors) }\end{array}$ & Pools & $\begin{array}{c}\text { Pools with positive } \\
\text { progeny }\end{array}$ \\
\hline \hline MG QF2 driver plasmid & phsp-pBac & 440 & 35 & 17 & P1 and P2 \\
\hline SG QF2 driver plasmid & phsp-pBac & 500 & 19 & 8 & P1 and P5 \\
\hline $\begin{array}{l}\text { QUAS-MP2-Scorpine effector } \\
\text { plasmid }\end{array}$ & phsp-pBac & 547 & 43 & 22 & P1 and P2 \\
\hline
\end{tabular}

831 